\title{
Gender-specific impact of personal health parameters on individual brain aging in cognitively unimpaired elderly subjects
}

\section{Katja Franke ${ }^{1,2 *}$, Michael Ristow ${ }^{3,4}$, Christian Gaser $^{1,2}$ and The Alzheimer's Disease Neuroimaging Initiative ${ }^{\dagger}$}

\author{
1 Structural Brain Mapping Group, Department of Psychiatry, Jena University Hospital, Jena, Germany \\ 2 Department of Neurology, Jena University Hospital, Jena, Germany \\ ${ }^{3}$ Department of Human Nutrition, Friedrich Schiller-University Jena, Jena, Germany \\ ${ }^{4}$ Energy Metabolism Laboratory, ETH Zurich (Swiss Federal Institute of Technology), Schwerzenbach, Zürich, Switzerland
}

\section{Edited by:}

Claudia Perez-Cruz, Centro de Investigaciones y Estudios

Avanzados, Mexico

Reviewed by:

Claudia Perez-Cruz, Centro de Investigaciones y Estudios

Avanzados, Mexico

Hans J. Grabe, University of

Greifswald, Germany

\section{*Correspondence:}

Katja Franke, Structural Brain

Mapping Group, Department of

Psychiatry, University of Jena,

Jahnstr. 3, D-07743 Jena, Germany

e-mail:katja.franke@uni-jena.de

${ }^{\dagger}$ Data used in the preparation of this article were obtained from the Alzheimer's Disease Neuroimaging Initiative (ADNI) database (www.loni.ucla.edu/ADNI). As such, the investigators within the ADNI contributed to the design and implementation of ADNI and/or provided data but did not participate in analysis or writing of this report.

Complete listing of ADNI investigators available at http://www.Ioni.usc.edu/ADNI/Data/ ADNI_Authorship_List.pdf.
Aging alters brain structure and function. Personal health markers and modifiable lifestyle factors are related to individual brain aging as well as to the risk of developing Alzheimer's disease (AD). This study used a novel magnetic resonance imaging (MRI)-based biomarker to assess the effects of 17 health markers on individual brain aging in cognitively unimpaired elderly subjects. By employing kernel regression methods, the expression of normal brain-aging patterns forms the basis to estimate the brain age of a given new subject. If the estimated age is higher than the chronological age, a positive brain age gap estimation (BrainAGE) score indicates accelerated atrophy and is considered a risk factor for developing AD. Within this cross-sectional, multi-center study 228 cognitively unimpaired elderly subjects (118 males) completed an MRI at 1.5Tesla, physiological and blood parameter assessments. The multivariate regression model combining all measured parameters was capable of explaining $39 \%$ of BrainAGE variance in males $(p<0.001)$ and $32 \%$ in females $(p<0.01)$. Furthermore, markers of the metabolic syndrome as well as markers of liver and kidney functions were profoundly related to BrainAGE scores in males $(p<0.05)$. In females, markers of liver and kidney functions as well as supply of vitamin $\mathrm{B}_{12}$ were significantly related to BrainAGE $(p<0.05)$. In conclusion, in cognitively unimpaired elderly subjects several clinical markers of poor health were associated with subtle structural changes in the brain that reflect accelerated aging, whereas protective effects on brain aging were observed for markers of good health. Additionally, the relations between individual brain aging and miscellaneous health markers show gender-specific patterns. The BrainAGE approach may thus serve as a clinically relevant biomarker for the detection of subtly abnormal patterns of brain aging probably preceding cognitive decline and development of AD.

Keywords: aging, Alzheimer's disease (AD), BrainAGE, lifestyle, magnetic resonance imaging (MRI), voxel-based morphometry (VBM)

\section{INTRODUCTION}

The global prevalence of dementia is projected to rise sharply over the next decades. By 2050, 1 in 85 persons worldwide will be affected by Alzheimer's disease (AD), the most common form of dementia (Brookmeyer et al., 2007). Manifold pathological changes accumulate over many years or decades before cognitive decline occurs gradually, with dementia representing the final stage of the pathological cascade (Frisoni et al., 2010; Jack et al., 2010). These pathological changes include precocious and/or accelerated brain aging (Fotenos et al., 2008; Driscoll et al., 2009; Sluimer et al., 2009; Wang et al., 2009; Spulber et al., 2010; Clark et al., 2012). Recently, atrophic regions detected in AD patients were found to largely overlap with those regions showing a normal age-related decline in healthy control subjects (Dukart et al., 2011). Hence, early identification of neuroanatomical changes deviating from the normal age-related atrophy pattern has the potential to improve clinical outcomes in the disease course through early treatment or prophylaxis (Ashburner et al., 2003).

Though "healthy" brain aging has been found to follow highly coordinated and sequenced patterns of brain tissue loss and cerebrospinal fluid (CSF) expansion (Pfefferbaum et al., 1994; Good et al., 2001; Resnick et al., 2003; Raz and Rodrigue, 2006; Terribilli et al., 2011), multiple factors affect and modify those individual trajectories. Several markers of poor health and/or inappropriate lifestyle (including obesity, high cholesterol, nicotine and alcohol abuse, hypertension, diabetes, as well as elevated serum total homocysteine (tHcy) and lower levels of vitamin $\mathrm{B}_{12}$ ) have been related to the risk of accelerated brain atrophy, cognitive decline, and even dementia (Clarke et al., 1998, 2007; Ellinson et al., 2004; Clarke, 2006; Steele et al., 2007; Solfrizzi et al., 2008; Chen et al., 2009; Fitzpatrick et al., 2009; Debette et al., 2010; Oulhaj et al., 2010; Zylberstein et al., 2011). Furthermore, the combination of 
risk factors was found to further boost the risk (Luchsinger et al., 2005). Particularly, components of the metabolic syndrome, i.e., a higher body mass index (BMI), elevated cholesterol and fasting glucose levels, and a higher diastolic blood pressure (DBP), are associated with a greater rate of brain atrophy (Enzinger et al., 2005) as well as an increased risk of dementia (Middleton and Yaffe, 2009).

In contrast, a healthy and well-balanced lifestyle (including physical activity, normal body weight, smoking cessation, Mediterranean diet, and moderate alcohol intake) was shown to lower the risk of cognitive decline and dementia (Peters et al., 2008; Solfrizzi et al., 2008; Luchsinger and Gustafson, 2009; Scarmeas et al., 2009; Xu et al., 2009; Erickson et al., 2010; Feart et al., 2010; Frisardi et al., 2010; Gu et al., 2010; Nepal et al., 2010). Maintaining cardiovascular health in midlife was recently suggested to be the most promising strategy for preventing cognitive impairment and dementia in late life (Hughes and Ganguli, 2009).

Based on the widespread but well-ordered brain tissue loss that occurs with healthy aging into senescence (Good et al., 2001), we previously proposed a modeling approach to identify abnormal aging-related brain atrophy that may precede the onset of cognitive decline and clinical symptoms. We introduced a novel BrainAGE approach (Franke et al., 2010, 2012b) based on a database of single time-point structural magnetic resonance imaging (MRI) data that aggregates the complex, multidimensional aging patterns across the whole brain to one single value, i.e., the estimated brain age. The difference between estimated and true chronological age will reveal the individual brain age gap estimation (BrainAGE) score. Consequently, the BrainAGE score directly quantifies subtle deviations in "normal" age-related brain atrophy by analyzing only one standard MRI per subject, with positive BrainAGE scores indicating accelerated structural brain aging and negative BrainAGE scores indicating decelerated structural brain aging. Recent work has demonstrated that increased BrainAGE scores in subjects with mild cognitive impairment (MCI) related to an increased risk of converting to $\mathrm{AD}$, with each additional year in the baseline BrainAGE score being associated with a $10 \%$ greater risk of converting to $\mathrm{AD}$ within the next three years (Gaser et al., 2013). Furthermore, we observed profound relationships between BrainAGE, disease severity, prospective worsening of cognitive functions (Franke et al., 2012a), conversion to AD (Gaser et al., 2013), as well as diabetes mellitus type 2 (Franke et al., 2013).

In this study, we implemented the BrainAGE method to explore and quantify the effects of several physiological and clinical chemistry markers of personal health on individual BrainAGE scores in a subsample of cognitively unimpaired older adults from the Alzheimer's Disease Neuroimaging Initiative (ADNI) database. Since men and women were found to differ in basic aspects of their normal function, their experience of the same illness (Pinn, 2003), probable risk factors on individual brain aging were separately examined in men and women. We expect gender-specific patterns in the relations between BrainAGE scores and health markers. Additionally, we expect the combination of the most significant risk factors to be associated with an even greater effect on individual BrainAGE scores than each factor independently.

\section{METHODS}

\section{SUBJECTS}

To train the age estimation framework, we used $\mathrm{T}_{1}$-weighted MRI data of 561 healthy subjects (250 male) from the publicly accessible IXI cohort (http://www.brain-development.org; data downloaded in September 2011) aged 20-86 years [mean $(S D)=$ 48.6 (16.5) years], which were collected on three different scanners (Philips 1.5T, General Electric 1.5T, Philips 3.0T). For more sample details see Franke et al. (2010).

The current BrainAGE analyses were conducted using data obtained from the ADNI database (www.loni.ucla.edu/ADNI). ADNI utilizes the following diagnostic criteria to classify the subjects into (1) Normal subjects (NO): "Mini-Mental State Examination" (MMSE; Cockrell and Folstein, 1988; test range 030) scores between 24 and 30 (inclusive), a "Clinical Dementia Rating" (CDR; Morris, 1993; test range 0-3) score of 0, non-depressed, non-MCI, and non-demented; (2) MCI subjects: MMSE scores between 24 and 30 (inclusive), a memory complaint, objective memory loss measured by education adjusted scores on Wechsler Memory Scale Logical Memory II, a CDR score of 0.5 , absence of significant levels of impairment in other cognitive domains, essentially preserved activities of daily living, and an absence of dementia; (3) mild AD: MMSE scores between 20 and 26 (inclusive), CDR scores of 0.5 or 1.0 , and meeting NINCDS/ADRDA criteria for probable AD. Detailed description of the ADNI inclusion and exclusion criteria is available at http://www.adni-info.org/Scientists/Pdfs/ adniproceduresmanual12.pdf.

Adopting the diagnostic classification from ADNI, we included all subjects who (i) were diagnosed as NO (i.e., cognitively unimpaired) at their baseline visit; for whom (ii) MRI data (1.5T), and (iii) a battery of physiological and clinical chemistry parameters at baseline were available [i.e., albumin, alanin-aminotransferase (ALT), aspartat-aminotransferase (AST), systolic (SBP) and diastolic blood pressure (DBP), BMI, $\gamma$-glutamyltransferase (GGT), glucose, mean erythrocyte cell volume (MCV), thyroid stimulating hormone (TSH), triglycerides, total bilirubin, creatinine, tHcy, uric acid, cholesterol, vitamin $\mathrm{B}_{12}$; Table 1]. Exact procedures of collection and processing of the physiological, clinical chemistry and MRI data can be found in the "ADNI Procedures Manual" (http://www.adni-info.org/Scientists/Pdfs/ adniproceduresmanual12.pdf).

Thus, the male test sample consisted of 118 cognitively unimpaired men, aged $60-88$ years, with a mean age of $75.8 \pm 5.3$ years. Mean MMSE at baseline was $29.0 \pm 1.1$. The female test sample contained 110 cognitively unimpaired women, aged 6290 years, with a mean age of $76.1 \pm 4.8$ years. Mean MMSE at baseline was $29.2 \pm 0.9$. More cognitive test scores for baseline and available follow-up assessments are presented in Table 2. Additionally, a list of subjects included in this study is provided in Supplement 1.

\section{PREPROCESSING OF MRI DATA AND DATA REDUCTION}

As described in Franke et al. (2010), preprocessing of the T1weighted images was done using the SPM8 package (http:// www.fil.ion.ucl.ac.uk/spm/) and the VBM8 toolbox (http://dbm. neuro.uni-jena.de), running under MATLAB. All T1-weighted images were corrected for bias-field inhomogeneities, then 
Table 1 | Baseline characteristics of all measured physiological and clinical chemistry parameters in male and female test samples.

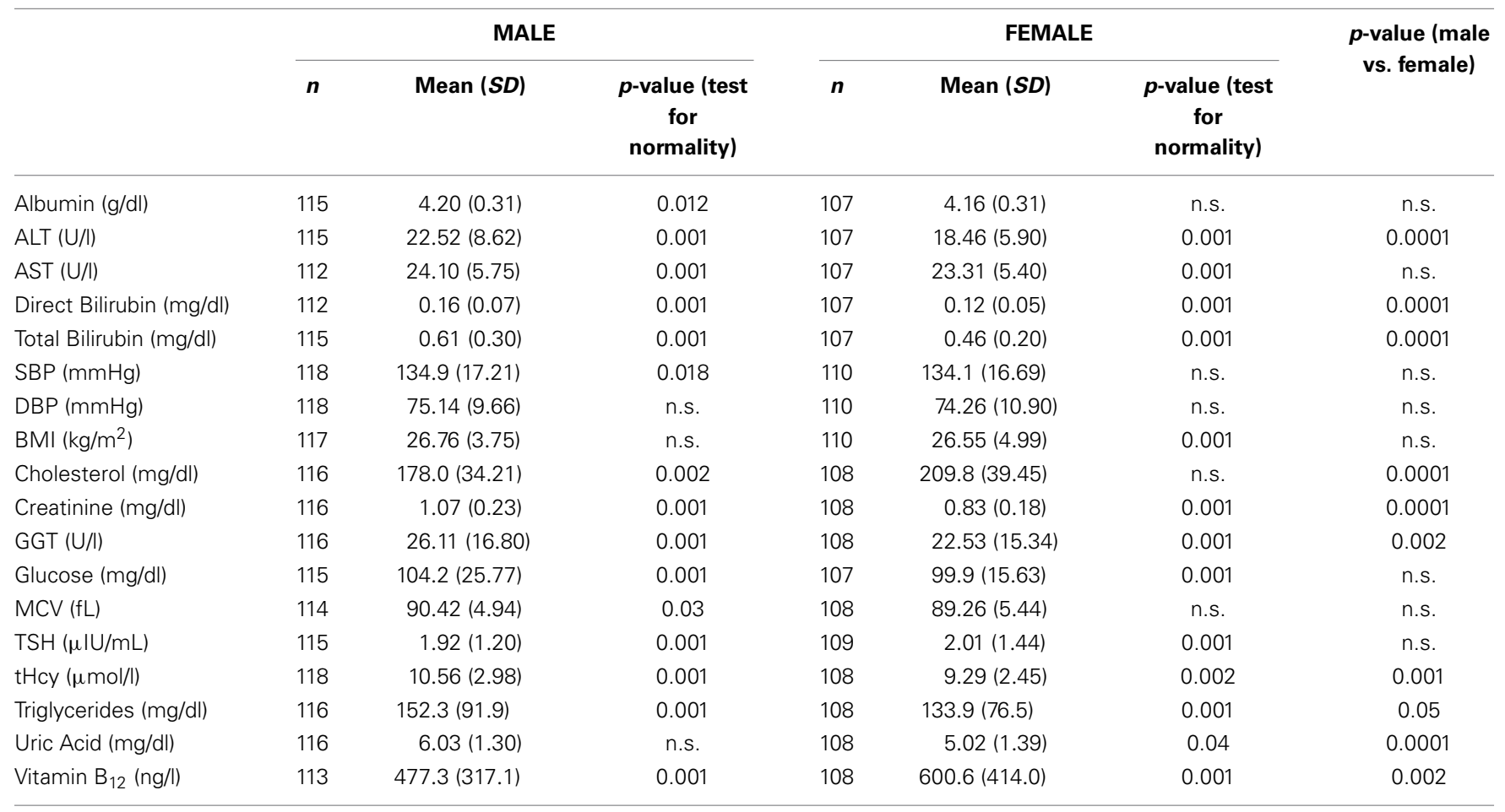

ALT, alanin-aminotransferase; AST, aspartat-aminotransferase; BMI, body mass index; DBP, diastolic blood pressure; GGT, $\gamma$-glutamyltransferase; MCV, mean erythrocyte cell volume; SBP, systolic blood pressure; TSH, thyroid stimulating hormone; $t$ Hcy, total homocysteine; n.s., not significant.

Table 2 | Cognitive characteristics in male and female test samples.

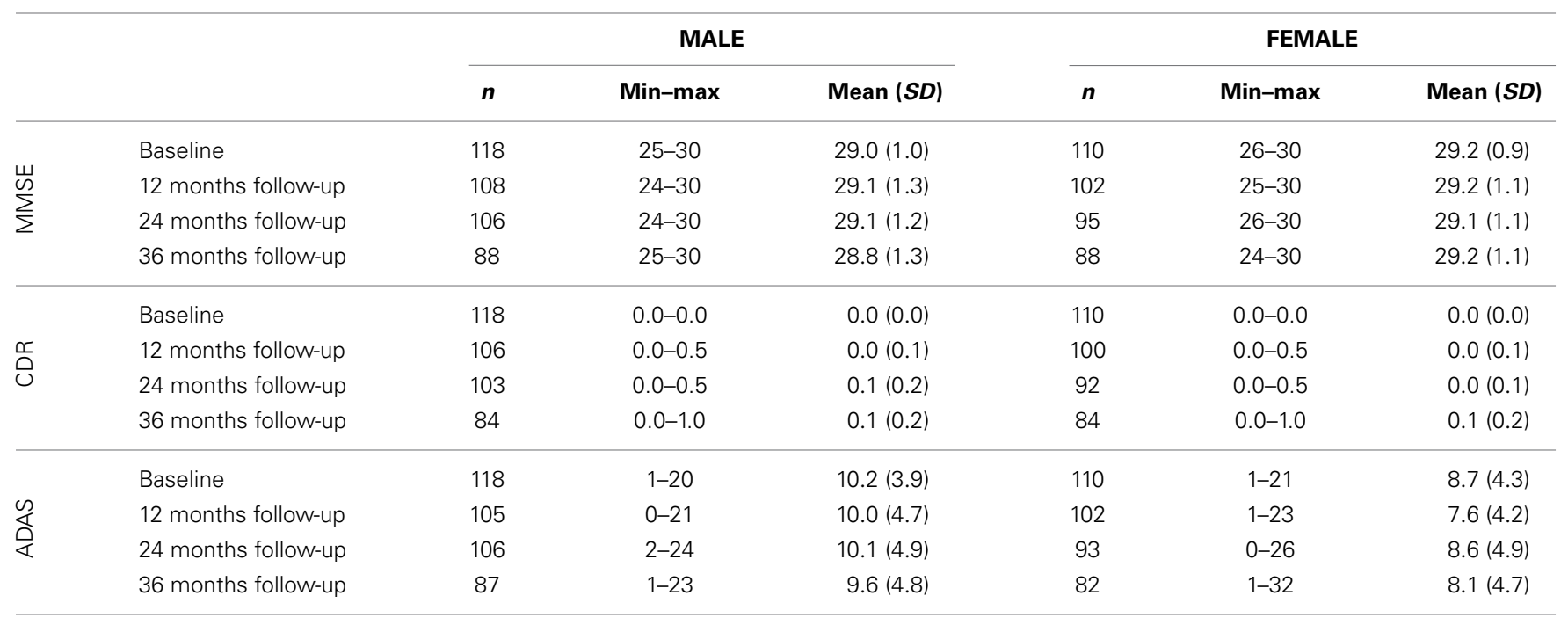

ADAS, Alzheimer's Disease Assessment Scale; CDR, Clinical Dementia Rating; MMSE, Mini-Mental State Examination.

spatially normalized and segmented into gray matter (GM), white matter (WM), and CSF within the same generative model (Ashburner and Friston, 2005). As recently described (Gaser, 2009), the segmentation procedure was further extended by accounting for partial volume effects (Tohka et al., 2004), applying adaptive maximum a posteriori estimations (Rajapakse et al., 1997), and using a hidden Markov Random Field model (Cuadra et al., 2005). After segmentation, only GM images were used. The images were processed with affine registration and smoothed with 8-mm full-width-at-half-maximum (FWHM) smoothing kernels. Spatial resolution was set to $8 \mathrm{~mm}$. Data reduction was performed by applying principal component analysis (PCA), utilizing the "Matlab Toolbox for Dimensionality Reduction" (http://ict.ewi. tudelft.nl/ lvandermaaten/Home.html). 


\section{AGE ESTIMATION FRAMEWORK}

The BrainAGE framework utilizes a machine-learning pattern recognition method, namely relevance vector regression (RVR; Tipping, 2001). It was recently developed to model healthy brain aging and subsequently estimate individual brain ages based on T1-weighted images (Franke et al., 2010). As suggested by Franke et al. (2010), the kernel was chosen to be a polynomial of degree 1 , since age estimation accuracy was shown to not improve when choosing non-linear kernels. Thus, parameter optimization during the training procedure was not necessary.

In general, the age regression model is trained with chronological age and preprocessed whole brain structural MRI data (as described in "Preprocessing of MRI Data and Data Reduction") of the training sample, resulting in a complex model of healthy brain aging (Figure 1A, left panel). Put in other words, the algorithm uses those whole-brain MRI data from the training sample that represent the prototypical examples within the specified regression task (i.e., healthy brain aging). Additionally, voxel-specific weights are calculated that represent the importance of each voxel within the specified regression task (i.e., healthy brain aging). For an illustration of the most important features (i.e., the importance of voxel locations for regression with age) that were used by the RVR to model normal brain aging and more detailed information please refer Franke et al. (2010).

Subsequently, the brain age of a test subject can be estimated using the individual tissue-classified MRI data (as described in "Preprocessing of MRI Data and Data Reduction"), aggregating the complex, multidimensional aging pattern across the whole brain into one single value (Figure 1A, right panel). In other words, all the voxels of the test subject's MRI data are weighted by applying the voxel-specific weighting matrix. Then, the brain age is calculated by applying the regression pattern of healthy brain aging and aggregating all voxel-wise information across the whole brain. The difference between estimated and chronological age will reveal the individual brain age gap estimation (BrainAGE) score, with positive values indicating accelerated structural brain aging and negative values indicating decelerated structural brain aging. Consequently, the BrainAGE score directly quantifies the amount of acceleration or deceleration of brain aging (Figure 1B). For example, if a 70 years old individual has a BrainAGE score of +5 years, this means that this individual shows the typical atrophy pattern of a 75 years old individual.

Recent work has demonstrated that this method provides reliable and stable estimates (Franke et al., 2012a). Specifically, the BrainAGE scores calculated from two shortly delayed scans on the same MRI scanner, as well as on separate $1.5 \mathrm{~T}$ and 3.0T scanners, produced intraclass correlation coefficients (ICC) of 0.93 and 0.90 , respectively.

Within this study, the BrainAGE framework was applied using the preprocessed GM images (as described in the section "Preprocessing of MRI Data and Data Reduction"). For training the model as well as for predicting individual brain ages, we used "The Spider" (http://www.kyb.mpg.de/bs/people/spider/ main.html), a freely available toolbox running under MATLAB.

\section{STATISTICAL ANALYSIS}

Descriptive statistics were used to summarize all variables. Physiological and clinical chemistry parameters as markers for of individual health status were compared between the male and female sample using analysis of variance (ANOVA) for normally distributed continuous variables or Kruskal-Wallis tests for variables that were not normally distributed. Normality was tested using Shapiro-Wilk tests. Since the ADNI database includes data from about 50 different study sites across the U.S. and Canada, the BrainAGE scores were compared between the several sites using analysis of variance (ANOVA) to test for probable site-specific effects.

The effect of gender within the relationships between BrainAGE and physiological and clinical chemistry parameters were investigated by performing analysis of covariance (ANCOVA). Each specific ANCOVA included all those subjects who were measured in each specific health and lifestyle parameter,
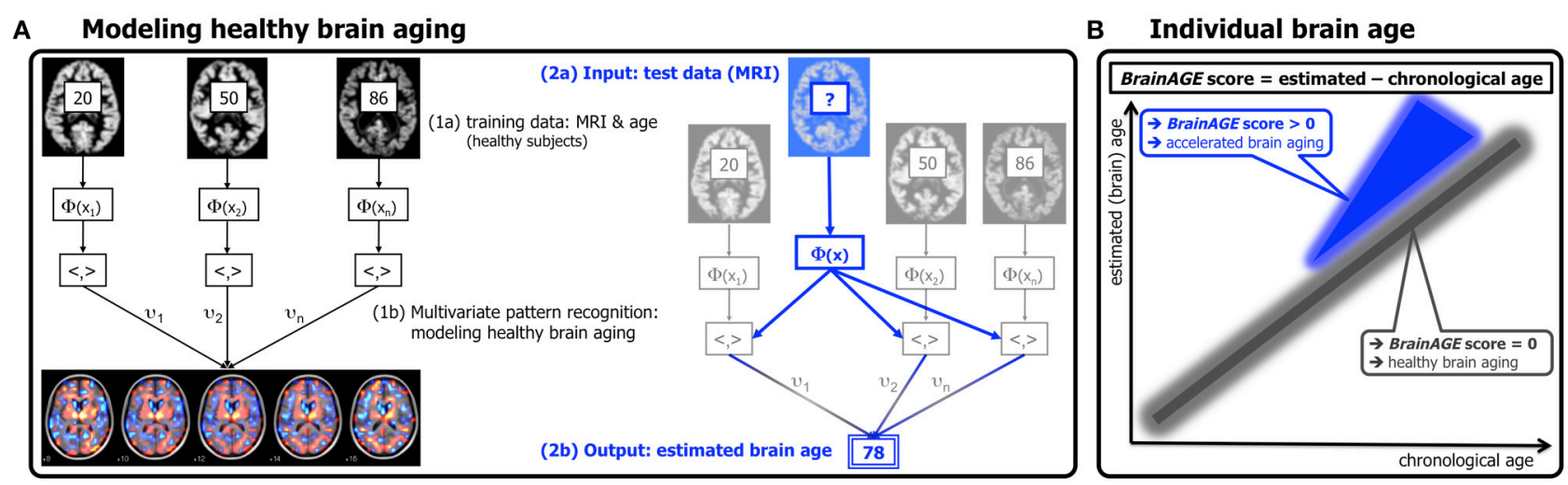

FIGURE 1 | Depiction of the BrainAGE concept. (A) The model of healthy brain aging is trained with the chronological age and preprocessed structural $\mathrm{MRI}$ data of a training sample (left; with an illustration of the most important voxel locations that were used by the age regression model). Subsequently, the individual brain ages of previously unseen test subjects are estimated, based on their MRI data (blue; picture modified from Schölkopf and Smola, 2002). (B) The difference between the estimated and chronological age results in the BrainAGE score, positive BrainAGE scores indicate accelerated brain aging. (Image reproduced from Franke et al. (2012a), with permission from Hogrefe Publishing, Bern). 
sub-grouped by gender. For each specific ANCOVA, the model fitted separate lines for the male and the female sample, thus allowing the intercept as well as the slopes to vary between both test samples.

Gender-specific effects of individual health parameters on BrainAGE were analyzed using linear regression models, specifically partial least squares (PLS). PLS included all subjects that were measured in all 17 physiological and clinical chemistry parameters, resulting in $n=107$ for the male sample (mean age $75.7 \pm 5.3$ years) and $n=104$ for the female sample (mean age $76.1 \pm 4.8$ years). However, as not all subjects were measured in all 17 physiological and clinical chemistry parameters, additional correlation analyses were performed for the most significant variables contributing to the variance in BrainAGE (based on the PLS variable weights) to further explore the relationships between BrainAGE and each of those physiological and clinical chemistry parameters. In order to control for covariates, Pearson's pairwise correlation for normally distributed variables or Spearman's for variables that are not normally distributed with adjustment for age and study site was used.

To quantify gender-specific effects of extremely low vs. extremely high levels in the most significant physiological and clinical chemistry parameters on BrainAGE, both test samples (i.e., male and female) were split up into quartiles for each of these clinical parameters. To illustrate the relationships between individual brain aging and extreme levels in each of these variables, the BrainAGE scores in the 1st quartile (lowest $25 \%$ of values) of each physiological and clinical chemistry parameter were tested against the BrainAGE scores in 4th quartile (highest $25 \%$ of values) of each physiological and clinical chemistry parameter, using Students' $t$-test for normally distributed parameters or Mann-Whitney test for those parameters that were not normally distributed. To control for multiple comparisons, Bonferroni-Holm correction (Holm, 1979) was applied, adjusting the $p$-value for the number of variables analyzed (i.e., 4; $p<0.05)$.

Additionally, to control for equal distribution in terms of chronological age within the 1st and 4th quartile groups as well as to explore the effects of extremely low vs. extremely high levels in the most significant physiological and clinical chemistry parameters on cognitive (i.e., Alzheimer's Disease Assessment Scale ADAS; Mohs and Cohen, 1988; Mohs, 1996) and disease severity scores (i.e., MMSE, CDR), Students' $t$-test for normally distributed parameters or Mann-Whitney test for those parameters that were not normally distributed were computed. Similar, Bonferroni-Holm-adjusted $p$-values were used to determine significance $(p<0.05)$.

Furthermore, the effect of combining the most significant variables (based on the PLS variable weights) on BrainAGE was explored in both test samples. Thereto, groups with "healthy" as well as "risky" clinical markers were formed. The groups with "healthy" clinical markers included all subjects who had values equal to or below the medians of the most significant physiological and clinical chemistry parameters (except for vitamin $B_{12}$, as higher values in vitamin $B_{12}$ are associated with more sufficient vitamin $B_{12}$ supply and therefore "better health"). The groups with "risky" clinical markers included all subjects who had values equal to or above the medians of the most significant physiological and clinical chemistry parameters (except for vitamin $B_{12}$, as lower values in vitamin $B_{12}$ are associated with an insufficient vitamin $B_{12}$ supply and therefore "poorer health"). Students' $t$-test was used to test these groups with combined "healthy" vs. combined "risky" clinical health marker values.

The Shapiro-Wilk test as well as PLS was performed using JMP 9.0 (www.jmp.com). All other testing was performed using MATLAB 7.11. (www.mathworks.com).

\section{RESULTS GROUP CHARACTERISTICS}

In the male as well as in the female test sample, the mean BrainAGE score was 0.0 years. There were no effects for scanning sites [male: $F_{(44,62)}=1.1, p=0.35$; female: $F_{(45,58)}=1.0, p=$ 0.43 ]. The mean values of the physiological and clinical chemistry data evaluated here are given in Table 1 . In the male test sample, only BMI, DBP, and uric acid were normally distributed. In the female test sample, albumin, SBP, DBP, cholesterol, and MCV were normally distributed. Men showed significantly higher parameter levels than women in ALT, Direct Bilirubin, Total Bilirubin, Creatinine, GGT, tHcy, Triglycerides, and Uric Acid, whereas women show significantly higher levels than men in Cholesterol and $\mathrm{B}_{12}$ (Table 1).

Table 3 | ANCOVA results for BrainAGE scores and health and lifestyle variables.

\begin{tabular}{|c|c|c|c|c|c|c|}
\hline & \multicolumn{6}{|c|}{ Model } \\
\hline & \multicolumn{2}{|c|}{ Gender } & \multicolumn{2}{|c|}{ Variable value } & \multicolumn{2}{|c|}{ Gender $x$ value } \\
\hline & $\boldsymbol{F}$ & $p$ & $\boldsymbol{F}$ & $p$ & $\boldsymbol{F}$ & $p$ \\
\hline Albumin (g/dl) & 0.03 & 0.87 & 0.47 & 0.49 & 0.17 & 0.68 \\
\hline ALT (U/I) & 0.16 & 0.69 & 0.58 & 0.45 & 3.90 & 0.05 \\
\hline AST (U/I) & 0.01 & 0.91 & 0.04 & 0.84 & 5.86 & 0.02 \\
\hline Direct Bilirubin (mg/dl) & 0.09 & 0.77 & 0.46 & 0.50 & 0.21 & 0.65 \\
\hline Total Bilirubin (mg/dl) & 0.56 & 0.45 & 3.74 & 0.06 & 1.23 & 0.27 \\
\hline $\mathrm{SBP}(\mathrm{mmHg})$ & 0.03 & 0.87 & 1.14 & 0.29 & 0.04 & 0.85 \\
\hline $\mathrm{DBP}(\mathrm{mmHg})$ & 0.06 & 0.80 & 6.40 & 0.01 & 0.85 & 0.36 \\
\hline BMI $\left(\mathrm{kg} / \mathrm{m}^{2}\right)$ & 0.05 & 0.82 & 18.81 & 0.0001 & 4.26 & 0.04 \\
\hline Cholesterol (mg/dl) & 0.00 & 0.99 & 0.39 & 0.53 & 0.81 & 0.37 \\
\hline Creatinine (mg/dl) & 0.71 & 0.40 & 1.47 & 0.23 & 0.01 & 0.93 \\
\hline GGT (U/I) & 0.44 & 0.51 & 12.49 & 0.001 & 0.00 & 0.98 \\
\hline Glucose (mg/dl) & 0.09 & 0.76 & 0.96 & 0.33 & 0.06 & 0.81 \\
\hline MCV (fL) & 0.03 & 0.86 & 0.68 & 0.41 & 0.24 & 0.62 \\
\hline TSH ( $\mu \mathrm{IU} / \mathrm{mL})$ & 0.08 & 0.77 & 3.11 & 0.08 & 0.22 & 0.64 \\
\hline $\mathrm{tHcy}(\mu \mathrm{mol} / \mathrm{l})$ & 0.17 & 0.68 & 3.82 & 0.05 & 0.05 & 0.82 \\
\hline Triglycerides (mg/dl) & 0.07 & 0.79 & 0.00 & 0.96 & 0.25 & 0.62 \\
\hline Uric Acid (mg/dl) & 1.42 & 0.23 & 6.98 & 0.01 & 2.52 & 0.11 \\
\hline Vitamin B12 (ng/l) & 0.23 & 0.63 & 1.7 & 0.19 & 2.47 & 0.12 \\
\hline
\end{tabular}

$A L T$, alanin-aminotransferase; $A S T$, aspartat-aminotransferase; BMI, body mass index; DBP, diastolic blood pressure; GGT, $\gamma$-glutamyltransferase; MCV, mean erythrocyte cell volume; SBP, systolic blood pressure; TSH, thyroid stimulating hormone; $t$ Hcy, total homocysteine; bold type = significant test results. 


\section{GENDER-SPECIFIC EFFECTS}

ANCOVAs were performed in order to investigate the effects of gender on the relationships between BrainAGE scores and all physiological and clinical chemistry data (Table 3). Within the whole test sample, BrainAGE scores varied with BMI $(p<$ $0.0001)$, DBP $(p<0.01)$, GGT $(p<0.001)$, and uric acid $(p<$ $0.01)$. Interactions with gender were found for ALT $(p<0.05)$, AST $(p<0.05)$, and BMI $(p<0.05)$. Thus, the effects of health parameters on BrainAGE show gender-specific patterns. Consequently, the following analyses were performed on males and females separately.

\section{MALE SAMPLE}

For men, when combining all measured physiological and clinical chemistry parameters in the PLS regression model, 39\% of variance within the BrainAGE score was attributed to the physiological and clinical chemistry parameters under consideration $\left(R^{2}=0.39, p<0.001\right)$. BMI, uric acid, GGT, and DBP contributed most to the variance in BrainAGE (Figure 2). More specifically, higher BrainAGE scores were significantly correlated to higher BMI $(r=0.35, p<0.001)$, increased DBP $(r=0.19$, $p<0.05)$, increased levels of GGT $(r=0.20, p<0.05)$, and

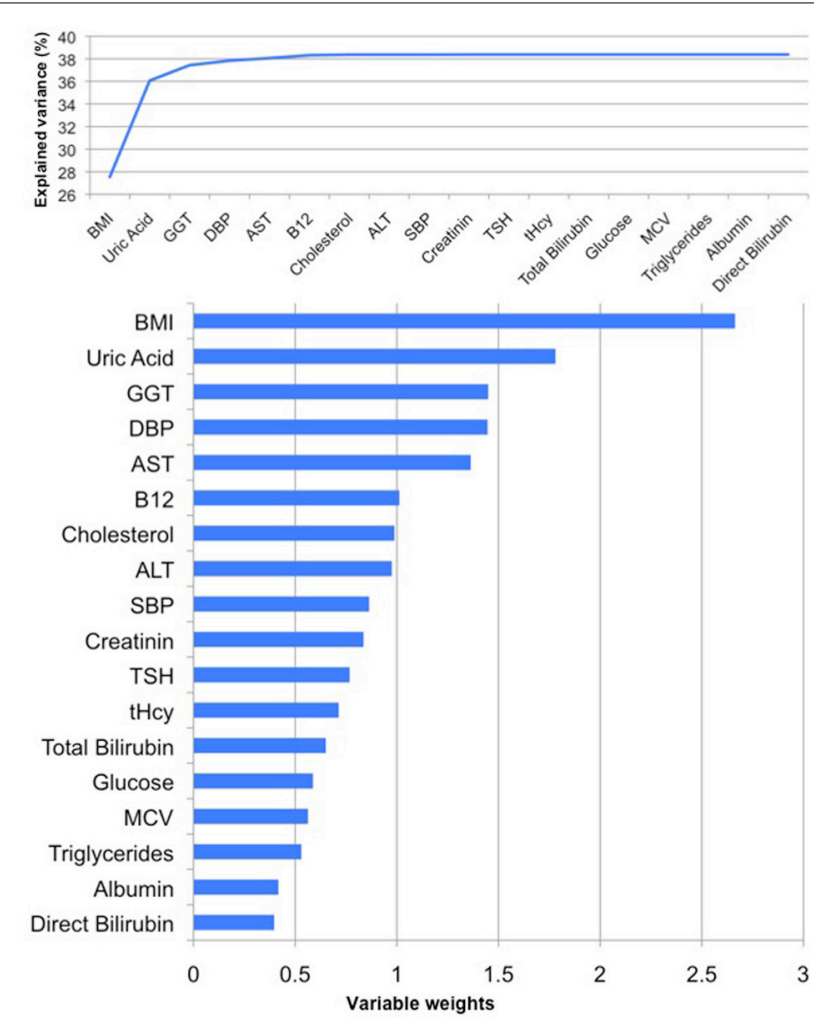

FIGURE 2 | PLS regression model of the male sample. When modeling the relationships between BrainAGE and health parameters, the PLS regression model explained $39 \%$ of variance in BrainAGE $(p<0.001)$ : BMI, uric acid, GGT, and DBP are the most significant physiological and clinical chemistry parameters as they added most to the explained variance in BrainAGE (top chart) and also showed the highest variable weights, i.e., the highest impact in the regression model (bottom chart). increased levels of uric acid $(r=0.25, p<0.01)$. This indicates a strong link between accelerated brain aging and elevated levels of these four parameters.

To quantify the effects of these most significant physiological and clinical chemistry parameters on BrainAGE, the BrainAGE scores of subjects with extremely low levels (i.e., 1st quartile group) were tested against the BrainAGE scores of subjects with extremely high levels (i.e., 4th quartile group) in each of those four parameters (Figure 3). In all four parameters higher values were related to higher BrainAGE scores, thus suggesting accelerated brain aging. The absolute difference of the mean BrainAGE scores in the lowest vs. the highest quartile group was 7.5 years for BMI $(p<0.001), 6.6$ years for DBP $(p<0.01), 7.5$ years for GGT $(p<0.01)$, and 5.6 years for uric acid $(p<0.05)$. All analyses survived the Bonferroni-Holm correction. Neither chronological age, nor cognitive scores at baseline and follow-up differed between 1st vs. 4 th quartile groups (Table 4).

Combining these four parameters, the effects on BrainAGE scores were compounded. More precisely, male subjects with values equal to or below the medians of BMI, DBP, GGT, and uric acid ("healthy" clinical markers; $n=9$ ) vs. male subjects with values equal to or above the medians of BMI, DBP, GGT, and uric acid ("risky" clinical markers; $n=14$ ) showed mean BrainAGE scores of -8.01 vs. 6.69 years, respectively $(p=0.015$; Figure 4). However, neither chronological age, nor cognitive scores at baseline and follow-up differed between both groups (Table 5).

Taken together, the results indicate a strong link between physiological and clinical health markers and structural brain aging in men, whereas no effects on cognitive scores could be found.

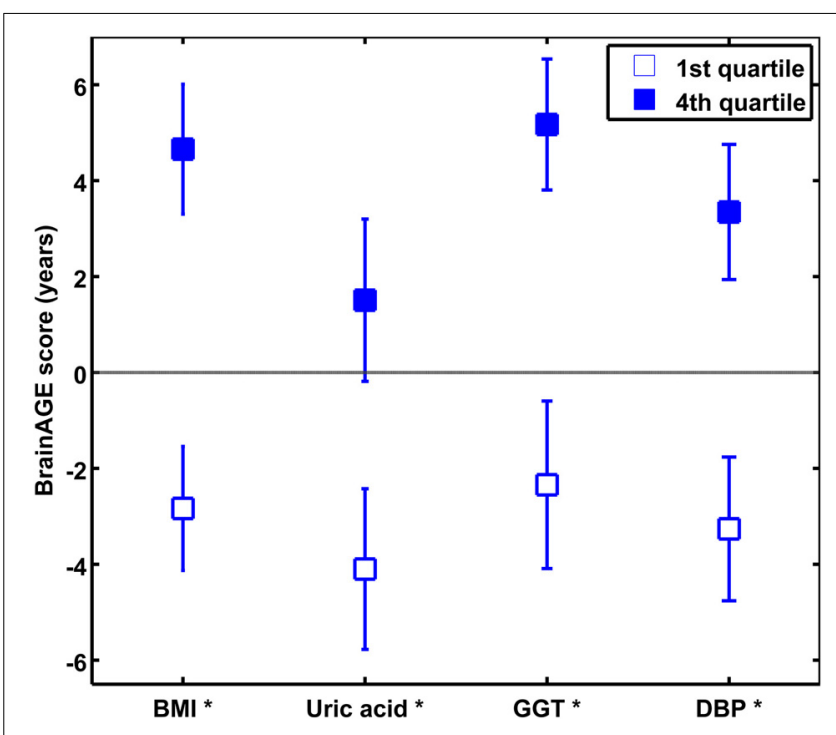

FIGURE 3 | The effects of extremely low vs. extremely high levels in clinical markers on BrainAGE in the male sample. Mean BrainAGE scores of male subjects in the 1st (plain) and the 4th quartiles (filled squares) of the most significant physiological and clinical chemistry parameters (i.e., BMI, uric acid, GGT, and DBP). Error bars depict the standard error of the mean (SEM). ${ }^{*} p<0.05$ after Bonferroni-Holm correction]. 
Table 4 | Means (SD) of BrainAGE scores, chronological age and cognitive scores in the 1st and 4th quartile groups of the four most significant physiological and clinical chemistry parameters within the male sample.

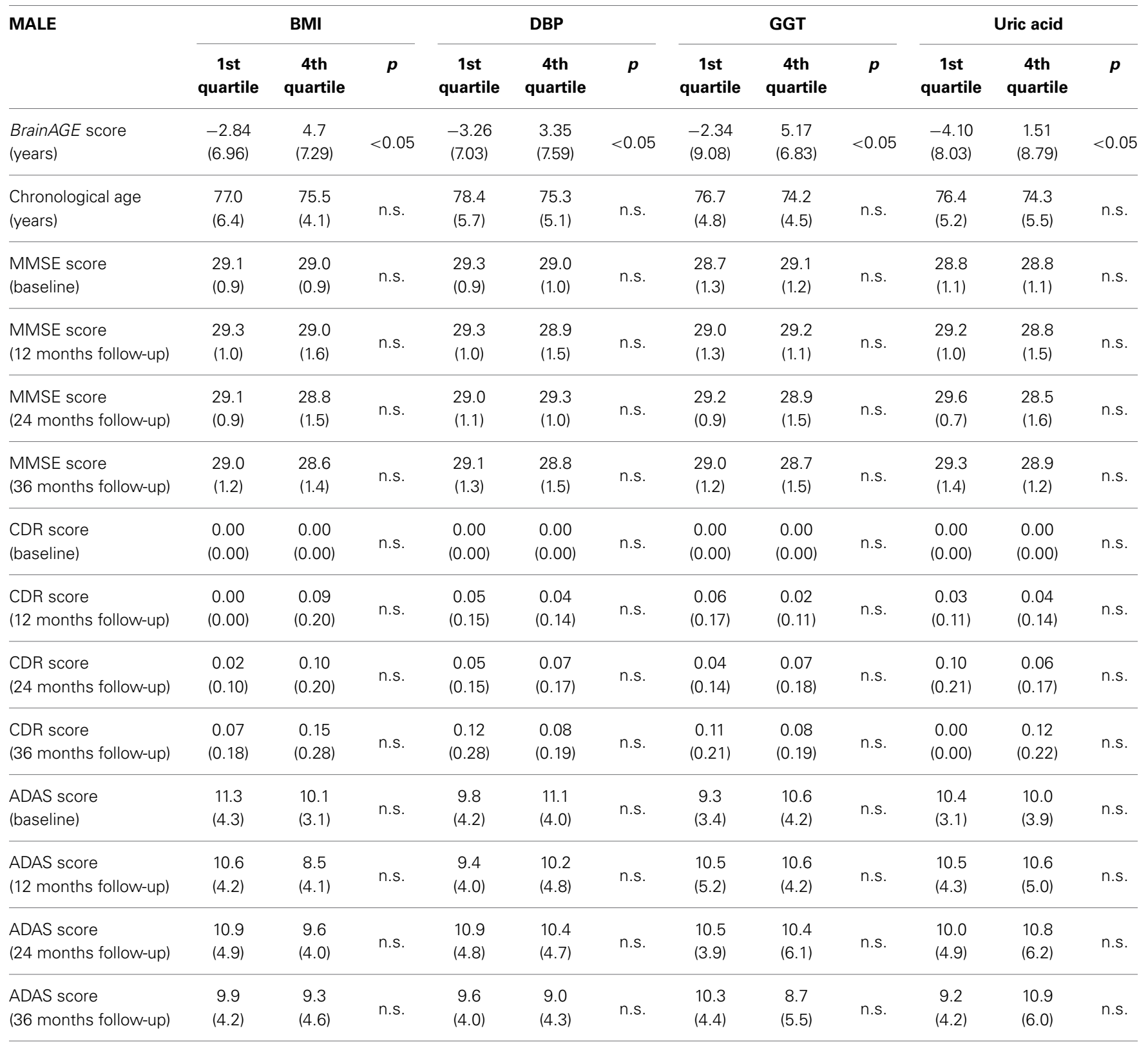

p-values of t-tests after Bonferroni-Holm correction, n.s., not significant.

$B M I$, body mass index; DBP, diastolic blood pressure; GGT, $\gamma$-glutamyltransferase; ADAS, Alzheimer's Disease Assessment Scale; CDR, Clinical Dementia Rating; MMSE, Mini-Mental State Examination.

\section{FEMALE SAMPLE}

For women, the PLS regression model combining all measured parameters was capable of explaining 32\% of BrainAGE variance $\left(R^{2}=0.32, p<0.01\right)$. As can be seen in Figure 5, GGT, AST, ALT, and vitamin $B_{12}$ contributed most to the variance in BrainAGE. More specifically, higher BrainAGE scores were significantly related to increased levels of GGT $(r=0.25, p<0.05)$, increased AST $(r=0.20, p<0.05)$ and ALT levels $(r=0.23, p<$ $0.05)$, and tended to be related to decreased levels of vitamin $B_{12}$ $(r=-0.17, p=0.08)$. Thus, the pattern of relationships between health and lifestyle markers and BrainAGE was different in the female and male samples.

Quantifying the effects of these four most significant physiological and clinical chemistry parameters on BrainAGE, the BrainAGE scores of subjects with extremely low levels (i.e., 1st quartile group) were tested against the BrainAGE scores of subjects with extremely high levels (i.e., 4th quartile group) in each of those four parameters (Figure 6). For GGT, AST, and ALT, higher parameter values were related to higher BrainAGE scores, thus suggesting accelerated brain aging. For vitamin $B_{12}$, higher 


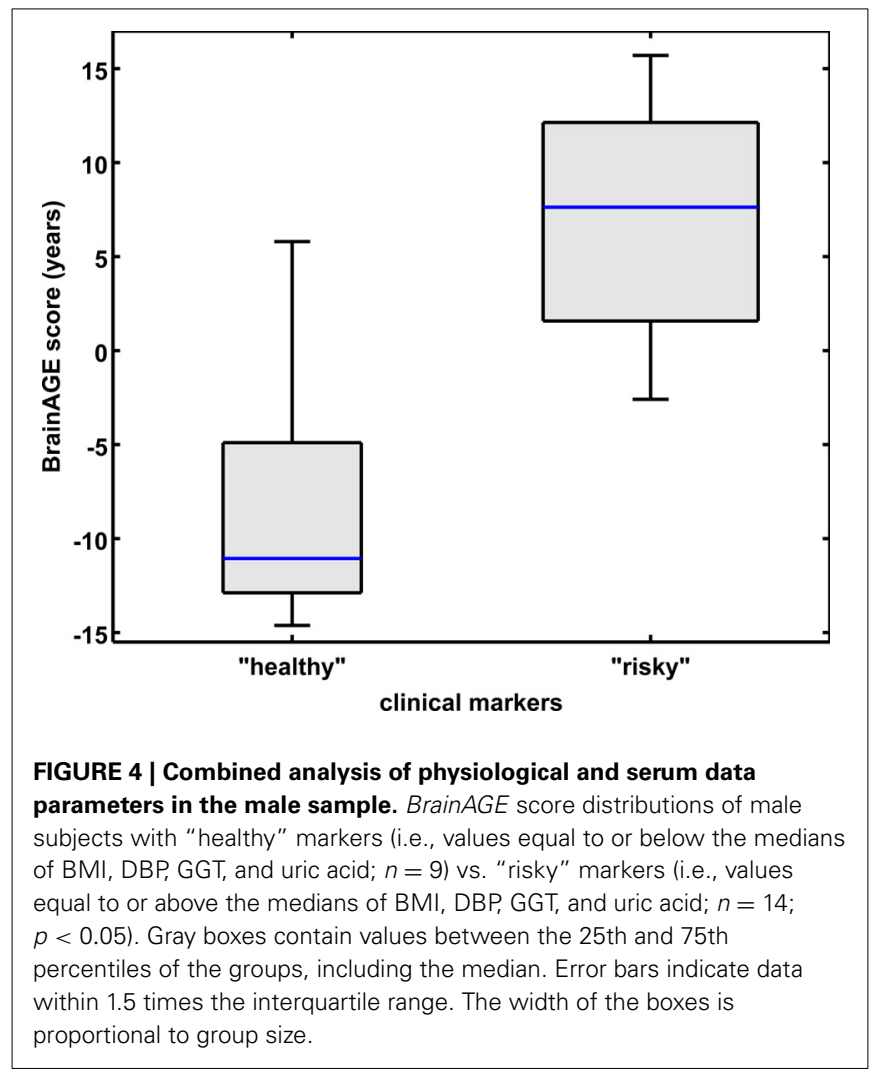

values were related to lower BrainAGE scores, thus suggesting a protective effect on brain aging. For GGT, the absolute difference of the mean BrainAGE scores was 6.1 years $(p<0.01)$; for AST, it resulted in 3.1 years $(p<0.10)$; for ALT in 5.1 years $(p<0.05)$; and for vitamin $\mathrm{B}_{12}$ in 4.8 years $(p<0.05)$. However, only GGT survived the Bonferroni-Holm correction. Neither chronological age, nor cognitive scores at baseline and follow-up differed between 1st vs. 4 th quartile groups (Table 6).

As already seen in the male sample, the effects on BrainAGE scores were compounded when combining those four parameters. More precisely, female subjects with values equal to or below the medians of GGT, AST, ALT, as well as values equal to or above the median of vitamin $\mathrm{B}_{12}$ ("healthy" clinical markers; $n=14$ ) vs. female subjects with values equal to or above the medians of GGT, AST, ALT, as well as values equal to or below the median of vitamin $\mathrm{B}_{12}$ ("risky" clinical markers; $n=13$ ) showed mean BrainAGE scores of -0.99 vs. 3.76 years, respectively ( $p=0.04$; Figure 7$)$. However, neither chronological age, nor cognitive scores at baseline and follow-up differed between both groups (Table 7).

Similar, but to a lesser extent as seen in the men's data, these results indicate a significant link between physiological and clinical health markers and structural brain aging in women, whereas no effects on cognitive scores could be found.

\section{DISCUSSION}

The scope of this study was the implementation of a novel MRIbased biomarker derived from the recently presented BrainAGE framework to quantify the effect of several common physiological
Table 5 | Means (SD) of BrainAGE scores, chronological age, and cognitive scores in male subjects with "healthy" clinical markers (i.e., values equal to or below the medians of BMI, DBP, GGT, and uric acid) vs. "risky" clinical markers (i.e., values equal to or above the medians of BMI, DBP, GGT, and uric acid).

\begin{tabular}{|c|c|c|c|}
\hline \multirow[t]{2}{*}{ Male } & \multicolumn{2}{|c|}{ Clinical markers } & \multirow[t]{2}{*}{$p$} \\
\hline & “healthy" & "risky" & \\
\hline $\begin{array}{l}\text { BrainAGE score } \\
\text { (years) }\end{array}$ & $\begin{array}{r}-8.01 \\
(7.11)\end{array}$ & $\begin{array}{l}6.69 \\
(6.48)\end{array}$ & $<0.05$ \\
\hline $\begin{array}{l}\text { Chronological age } \\
\text { (years) }\end{array}$ & $\begin{array}{l}75.6 \\
(5.5)\end{array}$ & $\begin{array}{l}73.0 \\
(5.2)\end{array}$ & n.s. \\
\hline $\begin{array}{l}\text { MMSE score } \\
\text { (baseline) }\end{array}$ & $\begin{array}{l}28.9 \\
(0.9)\end{array}$ & $\begin{array}{l}29.4 \\
(0.7)\end{array}$ & n.s. \\
\hline $\begin{array}{l}\text { MMSE score } \\
\text { (12 months follow-up) }\end{array}$ & $\begin{array}{l}29.7 \\
(0.7)\end{array}$ & $\begin{array}{l}29.8 \\
(0.6)\end{array}$ & n.s. \\
\hline $\begin{array}{l}\text { MMSE score } \\
\text { (24 months follow-up) }\end{array}$ & $\begin{array}{l}29.5 \\
(0.8)\end{array}$ & $\begin{array}{l}29.3 \\
(0.9)\end{array}$ & n.s. \\
\hline $\begin{array}{l}\text { MMSE score } \\
\text { (36 months follow-up) }\end{array}$ & $\begin{array}{l}29.4 \\
(0.8)\end{array}$ & $\begin{array}{l}28.6 \\
(1.7)\end{array}$ & n.s. \\
\hline $\begin{array}{l}\text { CDR score } \\
\text { (baseline) }\end{array}$ & $\begin{array}{c}0.00 \\
(0.00)\end{array}$ & $\begin{array}{c}0.00 \\
(0.00)\end{array}$ & n.s. \\
\hline $\begin{array}{l}\text { CDR score } \\
\text { (12 months follow-up) }\end{array}$ & $\begin{array}{c}0.06 \\
(0.17)\end{array}$ & $\begin{array}{c}0.04 \\
(0.13)\end{array}$ & n.s. \\
\hline $\begin{array}{l}\text { CDR score } \\
\text { ( } 24 \text { months follow-up) }\end{array}$ & $\begin{array}{c}0.12 \\
(0.23)\end{array}$ & $\begin{array}{c}0.00 \\
(0.00)\end{array}$ & n.s. \\
\hline $\begin{array}{l}\text { CDR score } \\
\text { (36 months follow-up) }\end{array}$ & $\begin{array}{c}0.00 \\
(0.00)\end{array}$ & $\begin{array}{c}0.04 \\
(0.14)\end{array}$ & n.s. \\
\hline $\begin{array}{l}\text { ADAS score } \\
\text { (baseline) }\end{array}$ & $\begin{array}{l}8.6 \\
(4.7)\end{array}$ & $\begin{array}{l}9.0 \\
(2.9)\end{array}$ & n.s. \\
\hline $\begin{array}{l}\text { ADAS score } \\
\text { (12 months follow-up) }\end{array}$ & $\begin{array}{l}9.8 \\
(4.4)\end{array}$ & $\begin{array}{c}7.9 \\
(4.1)\end{array}$ & n.s. \\
\hline $\begin{array}{l}\text { ADAS score } \\
\text { (24 months follow-up) }\end{array}$ & $\begin{array}{c}9.1 \\
(4.0)\end{array}$ & $\begin{array}{c}7.6 \\
(3.3)\end{array}$ & n.s. \\
\hline $\begin{array}{l}\text { ADAS score } \\
\text { ( } 36 \text { months follow-up) }\end{array}$ & $\begin{array}{c}8.6 \\
(4.8)\end{array}$ & $\begin{array}{l}8.8 \\
(3.4)\end{array}$ & n.s. \\
\hline
\end{tabular}

n.s., not significant.

ADAS, Alzheimer's Disease Assessment Scale; CDR, Clinical Dementia Rating; MMSE, Mini-Mental State Examination.

and clinical health markers on individual brain aging. Using structural MRI data, the BrainAGE approach aggregates the complex, multidimensional aging patterns across the whole brain to one single value (i.e., the BrainAGE score) and subsequently identifies pathological brain aging on an individual level. This method has been shown to accurately and reliably estimating the age of individual brains with minimal preprocessing and parameter optimization using anatomical MRI scans (Franke et al., 2010, 2012a). Additionally, higher BrainAGE scores were recently demonstrated to be related to measures of clinical disease 


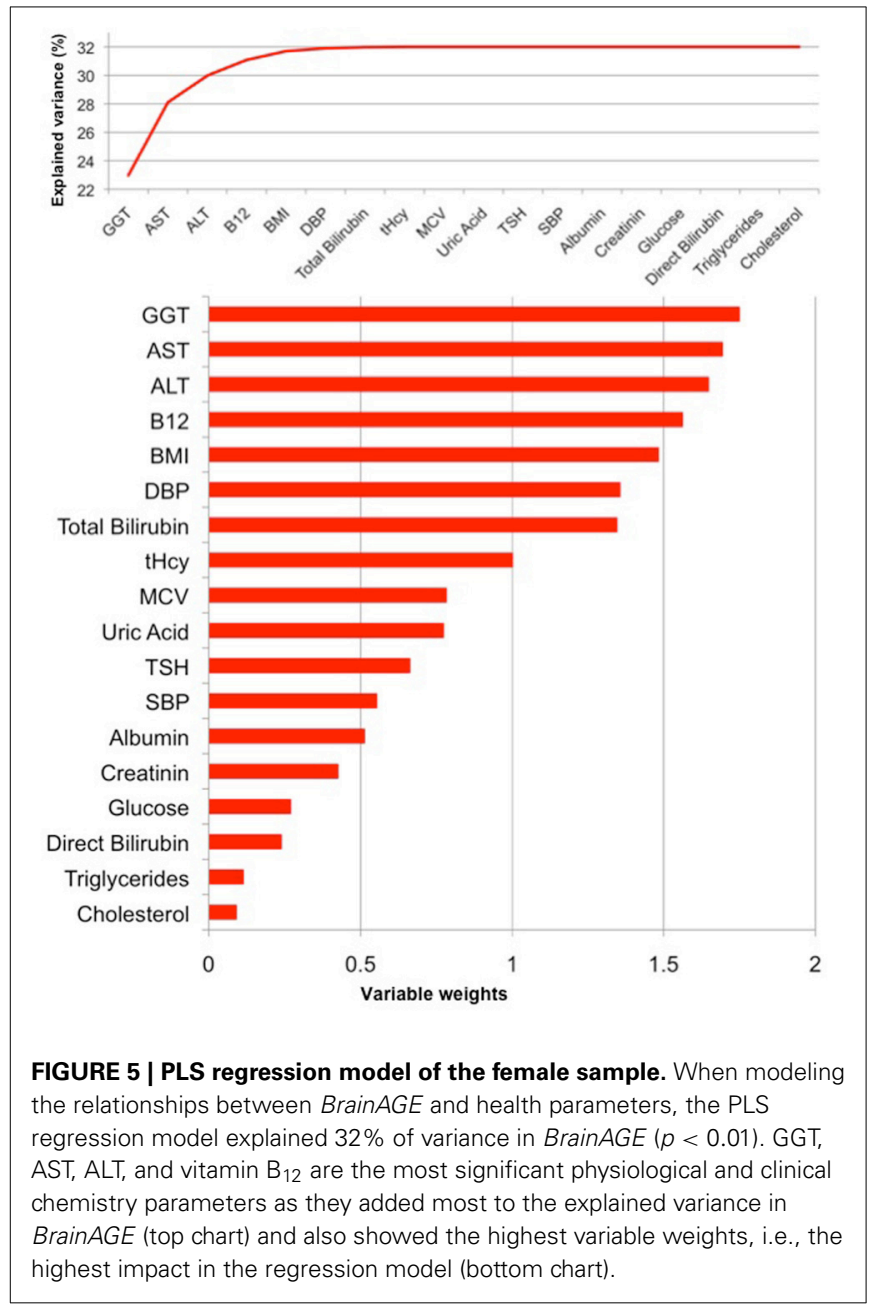

severity in $\mathrm{AD}$ patients, as well as prospective decline in cognitive functioning (Franke et al., 2012a) and conversion to AD (Gaser et al., 2013).

In this study, the BrainAGE approach was applied to a new sample, which included 110 female and 118 male cognitively unimpaired elderly subjects from the ADNI database. The results provide evidence that a number of physiological and clinical health parameters have significant effects on structural brain aging, hence possibly affecting the onset of dementia. Furthermore, the effects of health measures on BrainAGE showed gender-specific patterns.

In cognitively unimpaired elderly men the set of physiological and clinical health markers under consideration could explain $39 \%$ of variance in BrainAGE. More specifically, several markers of poor health were significantly related to higher BrainAGE scores, suggesting advanced brain atrophy. In particular, components of the metabolic syndrome (including elevated values in BMI, DBP, and uric acid) as well as markers of impaired liver function (including elevated levels of GGT and uric acid) were significantly related to increased BrainAGE scores of up to 9 years. This is consistent with previous studies that associated lower total brain volume as well as an increased risk of later dementia with a higher BMI and visceral adipose tissue at mid-life (Chen

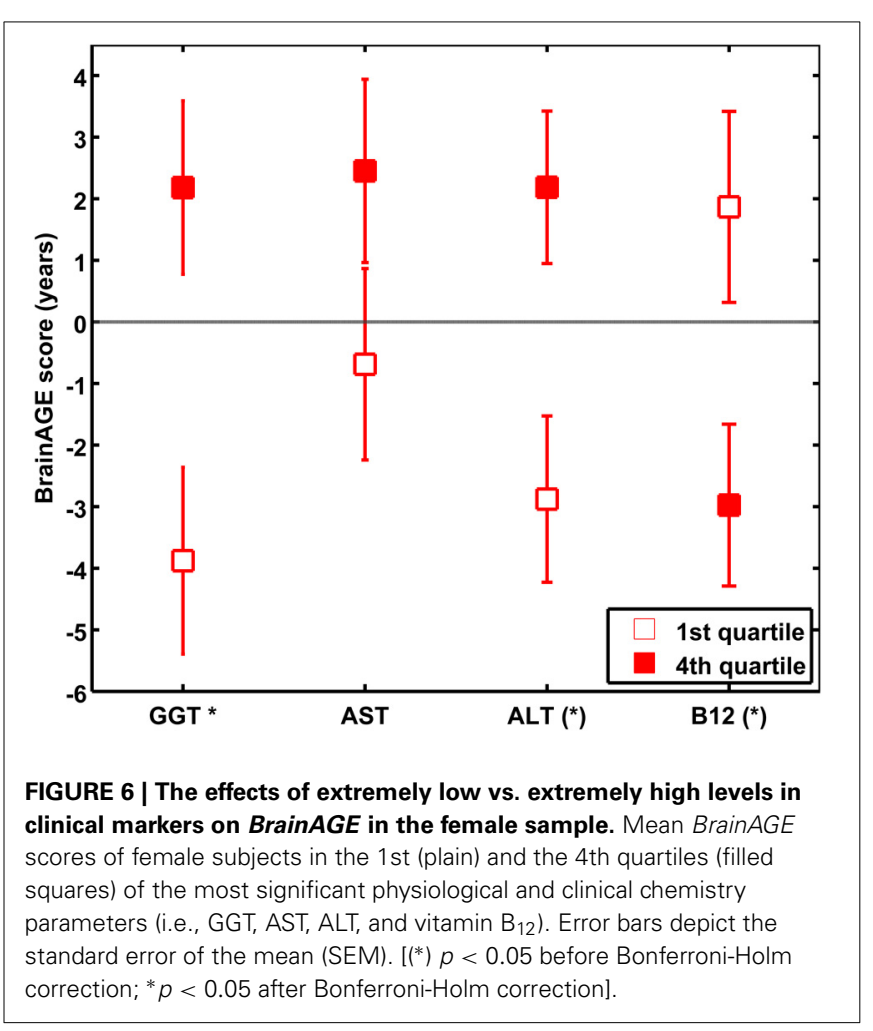

et al., 2009; Fitzpatrick et al., 2009; Debette et al., 2010) and the metabolic syndrome (Enzinger et al., 2005). However, those markers were neither related to cognitive scores at baseline, nor up to three years later.

In cognitively unimpaired elderly women, $32 \%$ of variance in BrainAGE was explained by the set of health and lifestyle markers under consideration. In particular, markers of liver and kidney functions (including ALT, AST, and GGT) as well as vitamin $\mathrm{B}_{12}$ levels were related to BrainAGE scores. Although it still remains uncertain how vitamin $B_{12}$ deficiency is linked to accelerated brain atrophy, cognitive decline, and dementia (McMahon et al., 2006; Aisen et al., 2008; Langan and Zawistoski, 2011), our results support recent studies, which suggested a neuroprotective role for vitamin $B_{12}$ (Clarke et al., 1998, 2007; Czapski et al., 2012; Morris, 2012; Morris et al., 2012; Douaud et al., 2013; Hinterberger and Fischer, 2013; Kim et al., 2013). This controversy in literature regarding the effects of vitamin $\mathrm{B}_{12}$ on brain structure and function may be due to the heterogeneity of study samples concerning age, gender, baseline cognition, and diagnosis etc. as well as a heterogeneity of utilized analysis methods. Further, we did not find any associations between BrainAGE and components of the metabolic syndrome in the female sample. These results are consistent with recent studies that also found gender-specific relationships between (lifestyle-related) health markers and GM atrophy (Taki et al., 2008) or even risk for AD (Chen et al., 2009).

Even more interesting, when combining the observed genderspecific risk parameters, the effects on BrainAGE were profoundly compounded in the male sample. This result is in line with Luchsinger et al. (2005), reporting an increased risk of AD with increased numbers of risk factors. However, in the female sample, 
Table 6 | Means (SD) of BrainAGE scores, chronological age and cognitive scores in the 1st and 4th quartile groups of the four most significant physiological and clinical chemistry parameters within the female sample.

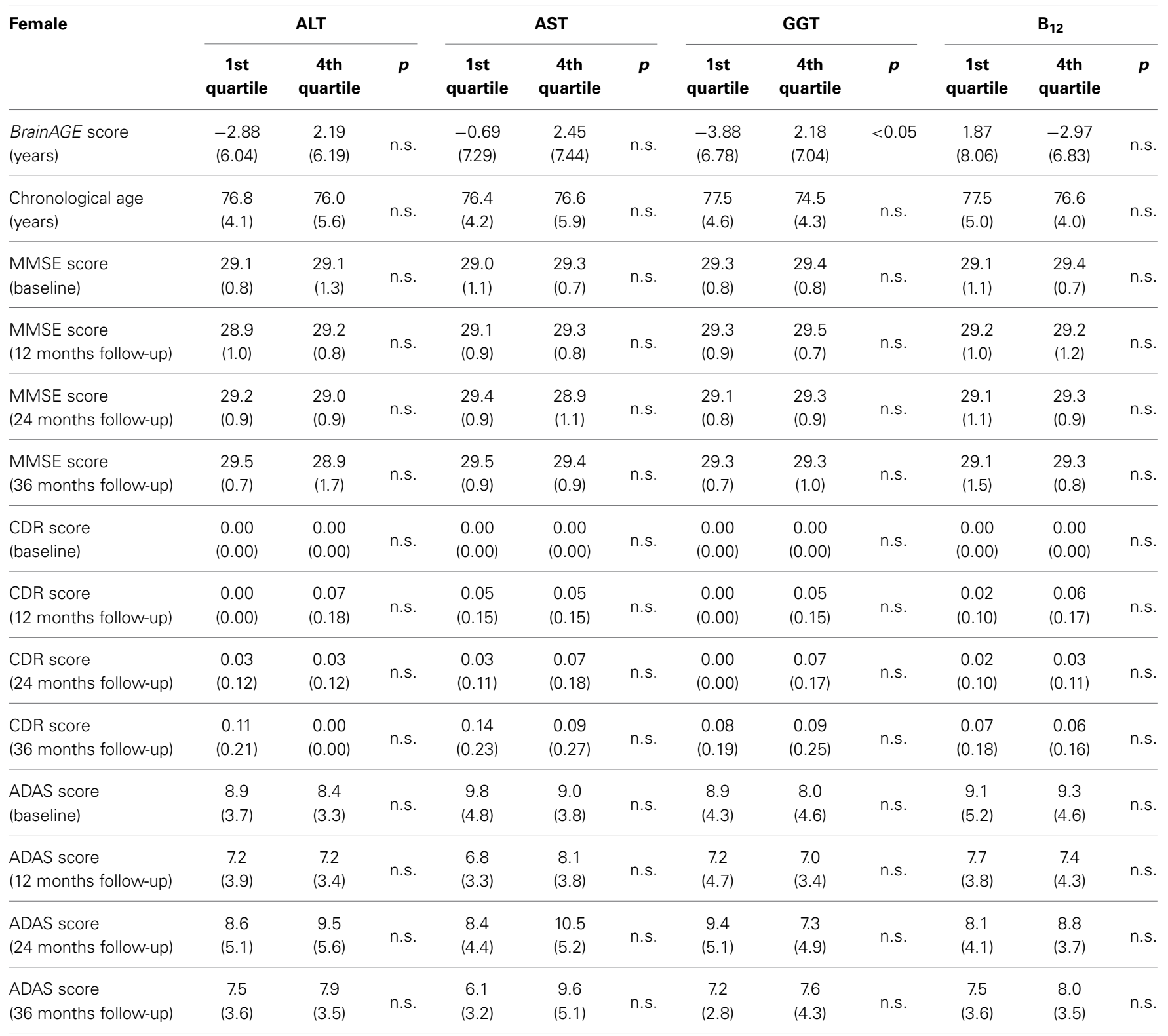

p-values of t-tests after Bonferroni-Holm correction; n.s., not significant.

ALT, alanin-aminotransferase; AST, aspartat-aminotransferase; GGT, $\gamma$-glutamyltransferase; ADAS, Alzheimer's Disease Assessment Scale; CDR, Clinical Dementia Rating; MMSE, Mini-Mental State Examination.

the compounding effect was much smaller, but still statistically significant. Additionally, the set of serum markers under consideration could explain 39\% of variance in BrainAGE in men, opposed to $32 \%$ in women. When analyzing men and women together, only components of the PLS pattern of the male sample were significantly related to increased BrainAGE scores (data not shown). Thus, the present study strongly suggests distinct genderspecific patterns of brain aging associated with certain health parameters, supporting the idea of the newly founded area of gender medicine (Pinn, 2003) that the phenomenon of aging as well as the prevention, detection, treatment, and outcome of illnesses affect men and women differently, including differences in basic aspects of their normal function and their experience of the same illness. Especially for $\mathrm{AD}$, it was suggested that the underlying mechanisms may be different in men and women (Grossi et al., 2005). Therefore, in the design of future studies, it should be imperative that there be enough women and men for appropriate gender-specific analyses (Azad et al., 2007).

Because this study was cross-sectional, it remains unclear whether certain health and lifestyle factors are cause or consequence of the associations found. Nevertheless, it strongly supports previously published results of personal lifestyle and overall 


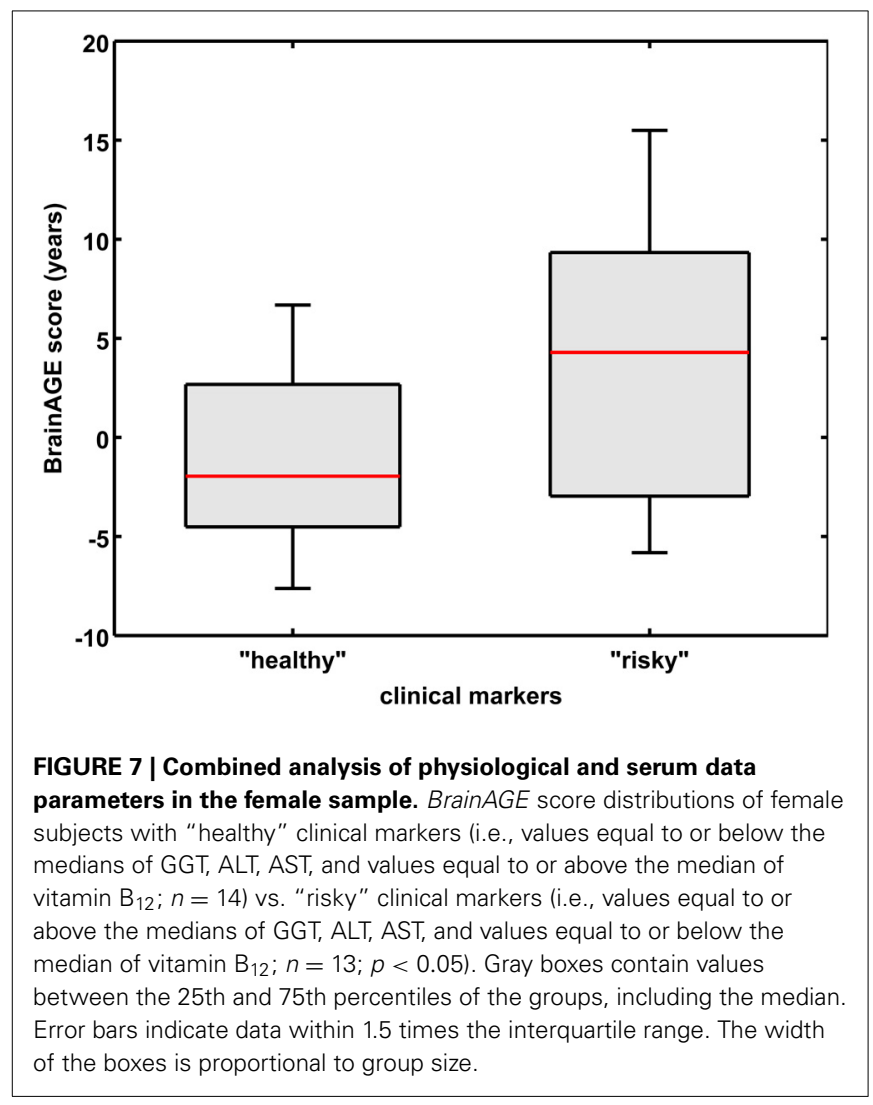

health being related to brain health, and extends this evidence by providing a small subset of serum markers that could explain nearly 40 percent of changes in BrainAGE.

Even more important, this study is the first that quantified the effects of several health markers on individual brain aging in terms of years. Relying on the assumption of AD being preceded by an acceleration in brain atrophy that resembles advanced aging (e.g., Driscoll et al., 2009; Cao et al., 2010; Spulber et al., 2010; Dukart et al., 2011; Jones et al., 2011; Saetre et al., 2011), subjects with increased BrainAGE scores are supposed to have a greater risk for conversion to $\mathrm{AD}$. However, the acceleration of spatiotemporal brain atrophy might only be seen in subjects in a preclinical stage, while in AD patients additional diseasespecific pathological changes are occurring. As recently shown by Dukart et al. (2013a,b), the magnitude of GM atrophy in healthy aging was comparable to the GM atrophy associated with increasing $\mathrm{AD}$ symptom severity within the age range of 50-80 years. Additionally, $\mathrm{AD}$ symptom severity was associated with age- and symptom severity-related add-ons in GM atrophy to normal age-related atrophy. Thus, and as already shown in MCI and $\mathrm{AD}$ subjects (Franke et al., 2012a; Gaser et al., 2013), apparently cognitively unimpaired subjects showing accelerated brain aging may have a greater risk for prospective worsening of cognitive functions and conversion to AD. However, cognitive reserve, genetic status, education level, socioeconomic status or lifestyle may protect subjects from pathological brain aging or accelerated cognitive decline despite high BrainAGE scores (Snowdon, 2003; Fotenos et al., 2008; Chen et al., 2009; Querbes et al., 2009;
Table 7 | Means (SD) of BrainAGE scores, chronological age, and cognitive scores in female subjects with "healthy" clinical markers (i.e., values equal to or below the medians of GGT, ALT, AST, and values equal to or above the median of vitamin $B_{12}$ ) vs. "risky" clinical markers (i.e., values equal to or above the medians of GGT, ALT, AST, and values equal to or below the median of vitamin $B_{12}$ ).

\begin{tabular}{|c|c|c|c|}
\hline \multirow[t]{2}{*}{ Female } & \multicolumn{2}{|c|}{ Clinical markers } & \multirow[t]{2}{*}{$p$} \\
\hline & "healthy" & "risky" & \\
\hline $\begin{array}{l}\text { BrainAGE score } \\
\text { (years) }\end{array}$ & $\begin{array}{l}-0.99 \\
(4.54)\end{array}$ & $\begin{array}{l}3.76 \\
(6.89)\end{array}$ & $<0.05$ \\
\hline $\begin{array}{l}\text { Chronological age } \\
\text { (years) }\end{array}$ & $\begin{array}{l}76.9 \\
(4.0)\end{array}$ & $\begin{array}{l}76.6 \\
(4.7)\end{array}$ & n.s. \\
\hline $\begin{array}{l}\text { MMSE score } \\
\text { (baseline) }\end{array}$ & $\begin{array}{l}29.2 \\
(1.0)\end{array}$ & $\begin{array}{l}29.4 \\
(0.6)\end{array}$ & n.s. \\
\hline $\begin{array}{l}\text { MMSE score } \\
\text { (12 months follow-up) }\end{array}$ & $\begin{array}{l}29.1 \\
(1.2)\end{array}$ & $\begin{array}{l}29.3 \\
(0.8)\end{array}$ & n.s. \\
\hline $\begin{array}{l}\text { MMSE score } \\
\text { (24 months follow-up) }\end{array}$ & $\begin{array}{l}29.2 \\
(1.0)\end{array}$ & $\begin{array}{l}28.7 \\
(1.0)\end{array}$ & n.s. \\
\hline $\begin{array}{l}\text { MMSE score } \\
\text { ( } 36 \text { months follow-up) }\end{array}$ & $\begin{array}{l}29.5 \\
(0.7)\end{array}$ & $\begin{array}{l}28.8 \\
(1.8)\end{array}$ & n.s. \\
\hline $\begin{array}{l}\text { CDR score } \\
\text { (baseline) }\end{array}$ & $\begin{array}{l}0.00 \\
(0.00)\end{array}$ & $\begin{array}{c}0.00 \\
(0.00)\end{array}$ & n.s. \\
\hline $\begin{array}{l}\text { CDR score } \\
\text { (12 months follow-up) }\end{array}$ & $\begin{array}{c}0.04 \\
(0.14)\end{array}$ & $\begin{array}{l}0.05 \\
(0.15)\end{array}$ & n.s. \\
\hline $\begin{array}{l}\text { CDR score } \\
\text { ( } 24 \text { months follow-up) }\end{array}$ & $\begin{array}{c}0.00 \\
(0.00)\end{array}$ & $\begin{array}{l}0.09 \\
(0.20)\end{array}$ & n.s. \\
\hline $\begin{array}{l}\text { CDR score } \\
\text { (36 months follow-up) }\end{array}$ & $\begin{array}{l}0.06 \\
(0.17)\end{array}$ & $\begin{array}{c}0.15 \\
(0.34)\end{array}$ & n.s. \\
\hline $\begin{array}{l}\text { ADAS score } \\
\text { (baseline) }\end{array}$ & $\begin{array}{c}7.4 \\
(4.9)\end{array}$ & $\begin{array}{c}6.6 \\
(3.8)\end{array}$ & n.s. \\
\hline $\begin{array}{l}\text { ADAS score } \\
\text { (12 months follow-up) }\end{array}$ & $\begin{array}{c}7.0 \\
(4.0)\end{array}$ & $\begin{array}{l}5.6 \\
(3.4)\end{array}$ & n.s. \\
\hline $\begin{array}{l}\text { ADAS score } \\
\text { (24 months follow-up) }\end{array}$ & $\begin{array}{l}8.1 \\
(4.1)\end{array}$ & $\begin{array}{c}7.1 \\
(4.5)\end{array}$ & n.s. \\
\hline $\begin{array}{l}\text { ADAS score } \\
\text { (36 months follow-up) }\end{array}$ & $\begin{array}{c}6.6 \\
(4.2)\end{array}$ & $\begin{array}{c}6.9 \\
(5.0)\end{array}$ & n.s. \\
\hline
\end{tabular}

n.s., not significant.

ADAS, Alzheimer's Disease Assessment Scale; CDR, Clinical Dementia Rating; MMSE, Mini-Mental State Examination.

Mangialasche et al., 2013). Future work will disentangle age- and disease-specific influences on the estimation of BrainAGE score to subsequently account for those influences.

Furthermore and similarly to other studies using the ADNI data and adopting its diagnostic criteria, a proportion of our sample of apparently cognitively unimpaired subjects might have a preclinical $\mathrm{AD}$ pathology. In our samples, seven female and 8 male subjects showed baseline MMSE scores lower than 28, thus might have a preclinical AD pathology. However, excluding those 
subjects from the analyses did not change the results substantially (data not shown), i.e., the patterns of gender-specific risk parameters and its relations to BrainAGE remained the same. Further research is therefore needed to extend our results and explore the longitudinal relationships between individual brain aging and miscellaneous factors (e.g., lifestyle, cognitive reserve, genetic status) in a larger population-based sample. Furthermore, the relationship between the duration of exposure to risk factors and accelerated brain aging, and whether reversal of modifiable factors might decelerate the progression of brain aging, should be explored.

Although applying the BrainAGE method results in single global estimation scores of the individual "brain age", it accounts for the multidimensional aging pattern across all voxels in the brain. With correlations between chronological age and estimated brain age of $r=0.92$ in healthy adults, aged 20-86 years (Franke et al., 2010), and $r=0.93$ in healthy children and adolescents, aged 5-18 years (Franke et al., 2012b), the BrainAGE framework has proven to be a straightforward method to accurately and reliably estimate brain age with minimal preprocessing and parameter optimization. Most remarkably, although brain maturation in childhood as well as brain aging in late life comprise very complex, multidimensional, and highly variable processes (Good et al., 2001; Wilke et al., 2003; Lenroot and Giedd, 2006; Lebel and Beaulieu, 2011), the confidence intervals of estimated brain age did not change as a function of age (Franke et al., 2010, 2012b), underlining the potential of the approach to correctly capture the multidimensional characteristics of the different maturational and aging processes occurring in childhood and old age, respectively. Additionally, the BrainAGE method proved its ability to provide very stable and reliable estimates of brain agingeven across different scanners (Franke et al., 2012a). However, the BrainAGE framework only provides information on the magnitude of the deviation in "brain aging" from the normal aging process, but not which regions are affected and to what extent. Future work will focus on local quantitative assessment of all regional alterations in a single patient's brain to reveal information about the areas that cause the difference between estimated "brain age" and chronological age.

In summary, accelerated brain aging in cognitively unimpaired elderly subjects is related to several physiological and clinical markers of poor health, whereas a protective effect on brain aging is observed for markers of good health. Since accelerated brain atrophy was shown to precede cognitive impairment in MCI and AD (Frisoni et al., 2010; Jack et al., 2010), this study suggests that a good health, including a normal weight, appropriate liver and kidney functions, and sufficient supply of vitamin $\mathrm{B}_{12}$ (Steele et al., 2007; Solfrizzi et al., 2008), can prevent or at least slow down acceleration in brain aging and certain disease processes. However, gender-specific mechanisms should be taken care of in future studies.

As BrainAGE scores are calculated from a single T1-weighted MRI per subject, using processing techniques that can be fully automated with multi-center data, this approach may be easily implemented into clinical practice in order to encourage the identification of subtle, yet clinically-significant, changes in brain structure. With regards to health and lifestyle markers, the implications of this study may lead to a clinical tool that identifies people at risk of faster degradation of brain structure and function and potential risk for dementias, thus contributing to an early diagnosis of neurodegenerative diseases and facilitating early treatment or preventative interventions.

\section{ACKNOWLEDGMENTS}

We are grateful to Dr. Rachel Yotter and Dr. Alissa Winkler for their comments on the manuscript. This work was supported in part by the German Ministry for Education and Research (Bundesministerium für Bildung und Forschung - BMBF) [01EV0709 to Christian Gaser] and the European Community [FP7 HEALTH, Project 279281 (BrainAge) to Katja Franke]. Additionally, this work is supported by the European Foundation for the Study of Diabetes, and the Jena Centre for Systems Biology of Ageing (JenAge) funded by the BMBF [0315581 to Michael Ristow]. The sponsors had no role in the design and conduct of the study; collection, management, analysis, and interpretation of the data; and preparation, review, or approval of the manuscript. Data collection and sharing for this project was funded by the Alzheimer's Disease Neuroimaging Initiative (ADNI) (National Institutes of Health Grant U01 AG024904). ADNI is funded by the National Institute on Aging, the National Institute of Biomedical Imaging and Bioengineering, and through generous contributions from the following: Abbott, AstraZeneca AB, Bayer Schering Pharma AG, Bristol-Myers Squibb, Eisai Global Clinical Development, Elan Corporation, Genentech, GE Healthcare, GlaxoSmithKline, Innogenetics, Johnson and Johnson, Eli Lilly and Co., Medpace, Inc., Merck and Co., Inc., Novartis AG, Pfizer Inc., F. Hoffman-La Roche, Schering-Plough, Synarc, Inc., as well as non-profit partners the Alzheimer's Association and Alzheimer's Drug Discovery Foundation, with participation from the U.S. Food and Drug Administration. Private sector contributions to ADNI are facilitated by the Foundation for the National Institutes of Health (www.fnih.org). The grantee organization is the Northern California Institute for Research and Education, and the study is coordinated by the Alzheimer's Disease Cooperative Study at the University of California, San Diego. ADNI data are disseminated by the Laboratory for Neuro Imaging at the University of California, Los Angeles. This research was also supported by NIH grants P30 AG010129, K01 AG030514, and the Dana Foundation.

\section{SUPPLEMENTARY MATERIAL}

The Supplementary Material for this article can be found online at: http://www.frontiersin.org/journal/10.3389/fnagi.2014. 00094/abstract

\section{REFERENCES}

Aisen, P. S., Schneider, L. S., Sano, M., Diaz-Arrastia, R., Van Dyck, C. H., Weiner, M. F., et al. (2008). High-dose B vitamin supplementation and cognitive decline in Alzheimer disease: a randomized controlled trial. JAMA 300, 1774-1783. doi: 10.1001/jama.300.15.1774

Ashburner, J., Csernansky, J. G., Davatzikos, C., Fox, N. C., Frisoni, G. B., and Thompson, P. M. (2003). Computer-assisted imaging to assess brain structure in healthy and diseased brains. Lancet Neurol. 2, 79-88. doi: 10.1016/S14744422(03)00304-1

Ashburner, J., and Friston, K. J. (2005). Unified segmentation. Neuroimage 26, 839-851. doi: 10.1016/j.neuroimage.2005.02.018 
Azad, N. A., Al Bugami, M., and Loy-English, I. (2007). Gender differences in dementia risk factors. Gend. Med. 4, 120-129. doi: 10.1016/S15508579(07)80026-X

Brookmeyer, R., Johnson, E., Ziegler-Graham, K., and Arrighi, H. M. (2007). Forecasting the global burden of Alzheimer's disease. Alzheimers. Dement. 3, 186-191. doi: 10.1016/j.jalz.2007.04.381

Cao, K., Chen-Plotkin, A. S., Plotkin, J. B., and Wang, L. S. (2010). Age-correlated gene expression in normal and neurodegenerative human brain tissues. PLoS ONE 5:e13098. doi: 10.1371/journal.pone.0013098

Chen, J. H., Lin, K. P., and Chen, Y. C. (2009). Risk factors for dementia. J. Formosan Med. Assoc. 108, 754-764. doi: 10.1016/S0929-6646(09)60402-2

Clark, V. H., Resnick, S. M., Doshi, J., Beason-Held, L. L., Zhou, Y., Ferucci, L., et al. (2012). Longitudinal imaging pattern analysis (SPARECD index) detects early structural and functional changes before cognitive decline in healthy older adults. Neurobiol. Aging 33, 2733-2745. doi: 10.1016/j.neurobiolaging.2012.01.010

Clarke, R. (2006). Vitamin B12, folic acid, and the prevention of dementia. N. Engl. J. Med. 354, 2817-2819. doi: 10.1056/NEJMe068086

Clarke, R., Birks, J., Nexo, E., Ueland, P. M., Schneede, J., Scott, J., et al. (2007). Low vitamin B-12 status and risk of cognitive decline in older adults. Am. J. Clin. Nutr. 86, 1384-1391.

Clarke, R., Smith, A. D., Jobst, K. A., Refsum, H., Sutton, L., and Ueland, P. M. (1998). Folate, vitamin B12, and serum total homocysteine levels in confirmed Alzheimer disease. Arch. Neurol. 55, 1449-1455. doi: 10.1001/archneur.55.11.1449

Cockrell, J. R., and Folstein, M. F. (1988). Mini-mental state examination (MMSE). Psychopharmacol. Bull. 24, 689-692.

Cuadra, M. B., Cammoun, L., Butz, T., Cuisenaire, O., and Thiran, J. P. (2005). Comparison and validation of tissue modelization and statistical classification methods in T1-weighted MR brain images. IEEE Trans. Med. Imaging 24, 1548-1565. doi: 10.1109/TMI.2005.857652

Czapski, G. A., Maruszak, A., Styczynska, M., Zekanowski, C., Safranow, K., and Strosznajder, J. B. (2012). Association between plasma biomarkers, CDK5 polymorphism and the risk of Alzheimer's disease. Acta Neurobiol. Exp. (Wars). 72, 397-411.

Debette, S., Beiser, A., Hoffmann, U., Decarli, C., O’donnell, C. J., Massaro, J. M., et al. (2010). Visceral fat is associated with lower brain volume in healthy middle-aged adults. Ann. Neurol. 68, 136-144. doi: 10.1002/ana.22062

Douaud, G., Refsum, H., De Jager, C. A., Jacoby, R., Nichols, T. E., Smith, S. M., et al. (2013). Preventing Alzheimer's disease-related gray matter atrophy by B-vitamin treatment. Proc. Natl. Acad. Sci. U.S.A. 110, 9523-9528. doi: 10.1073/pnas.1301816110

Driscoll, I., Davatzikos, C., An, Y., Wu, X., Shen, D., Kraut, M., et al. (2009). Longitudinal pattern of regional brain volume change differentiates normal aging from MCI. Neurology 72, 1906-1913. doi: 10.1212/WNL.0b013e3181 a 82634

Dukart, J., Kherif, F., Mueller, K., Adaszewski, S., Schroeter, M. L., Frackowiak, R. S., et al. (2013a). Generative FDG-PET and MRI model of aging and disease progression in Alzheimer's disease. PLoS Comput. Biol. 9:e1002987. doi: 10.1371/journal.pcbi.1002987

Dukart, J., Mueller, K., Villringer, A., Kherif, F., Draganski, B., Frackowiak, R., et al. (2013b). Relationship between imaging biomarkers, age, progression and symptom severity in Alzheimer's disease. Neuroimage Clin 3, 84-94. doi: 10.1016/j.nicl.2013.07.005

Dukart, J., Schroeter, M. L., and Mueller, K. (2011). Age correction in dementia-matching to a healthy brain. PLoS ONE 6:e22193. doi: 10.1371/journal.pone.0022193

Ellinson, M., Thomas, J., and Patterson, A. (2004). A critical evaluation of the relationship between serum vitamin $\mathrm{B}$, folate and total homocysteine with cognitive impairment in the elderly. J. Hum. Nutr. Diet. 17, 371-383. doi: 10.1111/j.1365-277X.2004.00532.x

Enzinger, C., Fazekas, F., Matthews, P. M., Ropele, S., Schmidt, H., Smith, S., et al. (2005). Risk factors for progression of brain atrophy in aging: six-year follow-up of normal subjects. Neurology 64, 1704-1711. doi: 10.1212/01.WNL.0000161871.83614.BB

Erickson, K. I., Raji, C. A., Lopez, O. L., Becker, J. T., Rosano, C., Newman, A. B., et al. (2010). Physical activity predicts gray matter volume in late adulthood: the Cardiovascular Health Study. Neurology 75, 1415-1422. doi: 10.1212/WNL.0b013e3181f88359
Feart, C., Samieri, C., and Barberger-Gateau, P. (2010). Mediterranean diet and cognitive function in older adults. Curr. Opin. Clin. Nutr. Metab. Care 13, 14-18. doi: 10.1097/MCO.0b013e3283331fe4

Fitzpatrick, A. L., Kuller, L. H., Lopez, O. L., Diehr, P., O’Meara, E. S., Longstreth, W. T., et al. (2009). Midlife and late-life obesity and the risk of dementia: cardiovascular health study. Arch. Neurol. 66, 336-342. doi: 10.1001/archneurol.2008.582

Fotenos, A. F., Mintun, M. A., Snyder, A. Z., Morris, J. C., and Buckner, R. L. (2008). Brain volume decline in aging: evidence for a relation between socioeconomic status, preclinical Alzheimer disease, and reserve. Arch. Neurol. 65, 113-120. doi: 10.1001/archneurol.2007.27

Franke, K., Gaser, C., and For the Alzheimer's Disease Neuroimaging Initiative. (2012a). Longitudinal changes in individual BrainAGE in healthy aging, mild cognitive impairment, and Alzheimer's disease. GeroPsych J. Gerontopsychol. Geriatr. Psychiatry 25, 235-245. doi: 10.1024/1662-9647/a000074

Franke, K., Gaser, C., Manor, B., and Novak, V. (2013). Advanced BrainAGE in older adults with type 2 diabetes mellitus. Front. Aging Neurosci. 5:90. doi: 10.3389/fnagi.2013.00090

Franke, K., Luders, E., May, A., Wilke, M., and Gaser, C. (2012b). Brain maturation: predicting individual BrainAGE in children and adolescents using structural MRI. Neuroimage 63, 1305-1312. doi: 10.1016/j.neuroimage.2012.08.001

Franke, K., Ziegler, G., Klöppel, S., Gaser, C., and The Alzheimer's Disease Neuroimaging Initiative (2010). Estimating the age of healthy subjects from T1-weighted MRI scans using kernel methods: exploring the influence of various parameters. Neuroimage 50, 883-892. doi: 10.1016/j.neuroimage. 2010.01.005

Frisardi, V., Panza, F., Seripa, D., Imbimbo, B. P., Vendemiale, G., Pilotto, A., et al. (2010). Nutraceutical properties of Mediterranean diet and cognitive decline: possible underlying mechanisms. J. Alzheimers. Dis. 22, 715-740. doi: 10.3233/JAD-2010-100942

Frisoni, G. B., Fox, N. C., Jack, C. R. Jr., Scheltens, P., and Thompson, P. M. (2010). The clinical use of structural MRI in Alzheimer disease. Nat. Rev. Neurol. 6, 67-77. doi: 10.1038/nrneurol.2009.215

Gaser, C. (2009). Partial volume segmentation with Adaptive Maximum a Posteriori (MAP) approach. Neuroimage 47, S121. doi: 10.1016/S10538119(09)71151-6

Gaser, C., Franke, K., Kloppel, S., Koutsouleris, N., and Sauer, H. (2013). BrainAGE in mild cognitive impaired patients: predicting the conversion to Alzheimer's disease. PLoS ONE 8:e67346. doi: 10.1371/journal.pone.0067346

Good, C. D., Johnsrude, I. S., Ashburner, J., Henson, R. N., Friston, K. J., and Frackowiak, R. S. (2001). A voxel-based morphometric study of ageing in 465 normal adult human brains. Neuroimage 14, 21-36. doi: 10.1006/nimg.2001.0786

Grossi, E., Massini, G., Buscema, M., Savare, R., and Maurelli, G. (2005). Two different Alzheimer diseases in men and women: clues from advanced neural networks and artificial intelligence. Gend. Med. 2, 106-117. doi: 10.1016/S15508579(05)80017-8

Gu, Y., Nieves, J. W., Stern, Y., Luchsinger, J. A., and Scarmeas, N. (2010). Food combination and Alzheimer disease risk: a protective diet. Arch. Neurol. 67, 699-706. doi: 10.1001/archneurol.2010.84

Hinterberger, M., and Fischer, P. (2013). Folate and Alzheimer: when time matters. J. Neural Transm. 120, 211-224. doi: 10.1007/s00702-012-0822-y

Holm, S. (1979). A simple sequentially rejective multiple test procedure. Scand. J. Stat. 6, 65-70.

Hughes, T. F., and Ganguli, M. (2009). Modifiable midlife risk factors for latelife cognitive impairment and dementia. Curr. Psychiatry Rev. 5, 73-92. doi: $10.2174 / 157340009788167347$

Jack, C. R. Jr., Knopman, D. S., Jagust, W. J., Shaw, L. M., Aisen, P. S., Weiner, M. W., et al. (2010). Hypothetical model of dynamic biomarkers of the Alzheimer's pathological cascade. Lancet Neurol. 9, 119-128. doi: 10.1016/S1474-4422(09) 70299-6

Jones, D. T., Machulda, M. M., Vemuri, P., McDade, E. M., Zeng, G., Senjem, M. L., et al. (2011). Age-related changes in the default mode network are more advanced in Alzheimer disease. Neurology 77, 1524-1531. doi: 10.1212/WNL.0b013e318233b33d

Kim, G., Kim, H., Kim, K. N., Son, J. I., Kim, S. Y., Tamura, T., et al. (2013). Relationship of cognitive function with $\mathrm{B}$ vitamin status, homocysteine, and tissue factor pathway inhibitor in cognitively impaired elderly: a cross-sectional survey. J. Alzheimers. Dis. 33, 853-862. doi: 10.3233/JAD-2012-121345 
Langan, R. C., and Zawistoski, K. J. (2011). Update on vitamin B12 deficiency. Am. Fam. Physician 83, 1425-1430.

Lebel, C., and Beaulieu, C. (2011). Longitudinal development of human brain wiring continues from childhood into adulthood. J. Neurosci. 31, 10937-10947. doi: 10.1523/JNEUROSCI.5302-10.2011

Lenroot, R. K., and Giedd, J. N. (2006). Brain development in children and adolescents: insights from anatomical magnetic resonance imaging. Neurosci. Biobehav. Rev. 30, 718-729. doi: 10.1016/j.neubiorev.2006.06.001

Luchsinger, J. A., and Gustafson, D. R. (2009). Adiposity and Alzheimer's disease. Curr. Opin. Clin. Nutr. Metab. Care 12, 15-21. doi: 10.1097/MCO.0b013e3283 $1 \mathrm{c} 8 \mathrm{c} 71$

Luchsinger, J. A., Reitz, C., Honig, L. S., Tang, M. X., Shea, S., and Mayeux, R. (2005). Aggregation of vascular risk factors and risk of incident Alzheimer disease. Neurology 65, 545-551. doi: 10.1212/01.wnl.0000172914.08967.dc

Mangialasche, F., Westman, E., Kivipelto, M., Muehlboeck, J. S., Cecchetti, R., Baglioni, M., et al. (2013). Classification and prediction of clinical diagnosis of Alzheimer's disease based on MRI and plasma measures of alpha/gamma-tocotrienols and gamma-tocopherol. J. Intern. Med. 273, 602-621. doi: 10.1111/joim. 12037

McMahon, J. A., Green, T. J., Skeaff, C. M., Knight, R. G., Mann, J. I., and Williams, S. M. (2006). A controlled trial of homocysteine lowering and cognitive performance. N. Engl. J. Med. 354, 2764-2772. doi: 10.1056/NEJMoa054025

Middleton, L. E., and Yaffe, K. (2009). Promising strategies for the prevention of dementia. Arch. Neurol. 66, 1210-1215. doi: 10.1001/archneurol.2009.201

Mohs, R. C. (1996). The Alzheimer's Disease Assessment Scale. Int. Psychogeriatr. 8, 195-203. doi: 10.1017/S1041610296002578

Mohs, R. C., and Cohen, L. (1988). Alzheimer's disease assessment scale (ADAS). Psychopharmacol. Bull. 24, 627-628.

Morris, J. C. (1993). The clinical dementia rating (CDR): current version and scoring rules. Neurology 43, 2412-2414. doi: 10.1212/WNL.43.11.2412-a

Morris, M. S. (2012). The role of B vitamins in preventing and treating cognitive impairment and decline. Adv. Nutr. 3, 801-812. doi: 10.3945/an.112.002535

Morris, M. S., Selhub, J., and Jacques, P. F. (2012). Vitamin B-12 and folate status in relation to decline in scores on the mini-mental state examination in the framingham heart study. J. Am. Geriatr. Soc. 60, 1457-1464. doi: $10.1111 / j .1532-5415.2012 .04076 . x$

Nepal, B., Brown, L., and Ranmuthugala, G. (2010). Modelling the impact of modifying lifestyle risk factors on dementia prevalence in Australian population aged 45 years and over, 2006-2051. Australas. J. Ageing 29, 111-116. doi: 10.1111/j.1741-6612.2010.00392.x

Oulhaj, A., Refsum, H., Beaumont, H., Williams, J., King, E., Jacoby, R., et al. (2010). Homocysteine as a predictor of cognitive decline in Alzheimer's disease. Int. J. Geriatr. Psychiatry 25, 82-90. doi: 10.1002/gps.2303

Peters, R., Poulter, R., Warner, J., Beckett, N., Burch, L., and Bulpitt, C. (2008). Smoking, dementia and cognitive decline in the elderly, a systematic review. BMC Geriatr. 8:36. doi: 10.1186/1471-2318-8-36

Pfefferbaum, A., Mathalon, D. H., Sullivan, E. V., Rawles, J. M., Zipursky, R. B., and Lim, K. O. (1994). A quantitative magnetic resonance imaging study of changes in brain morphology from infancy to late adulthood. Arch. Neurol. 51, 874-887. doi: 10.1001/archneur.1994.00540210046012

Pinn, V. W. (2003). Sex and gender factors in medical studies: implications for health and clinical practice. JAMA 289, 397-400. doi: 10.1001/jama.289.4.397

Querbes, O., Aubry, F., Pariente, J., Lotterie, J. A., Demonet, J. F., Duret, V., et al. (2009). Early diagnosis of Alzheimer's disease using cortical thickness: impact of cognitive reserve. Brain 132, 2036-2047. doi: 10.1093/brain/awp105

Rajapakse, J. C., Giedd, J. N., and Rapoport, J. L. (1997). Statistical approach to segmentation of single-channel cerebral MR images. IEEE Trans. Med. Imaging 16, 176-186. doi: 10.1109/42.563663

Raz, N., and Rodrigue, K. M. (2006). Differential aging of the brain: patterns, cognitive correlates and modifiers. Neurosci. Biobehav. Rev. 30, 730-748. doi: 10.1016/j.neubiorev.2006.07.001

Resnick, S. M., Pham, D. L., Kraut, M. A., Zonderman, A. B., and Davatzikos, C. (2003). Longitudinal magnetic resonance imaging studies of older adults: a shrinking brain. J. Neurosci. 23, 3295-3301.

Saetre, P., Jazin, E., and Emilsson, L. (2011). Age-related changes in gene expression are accelerated in Alzheimer's disease. Synapse 65, 971-974. doi: $10.1002 /$ syn.20933
Scarmeas, N., Luchsinger, J. A., Schupf, N., Brickman, A. M., Cosentino, S., Tang, M. X., et al. (2009). Physical activity, diet, and risk of Alzheimer disease. J. Am. Med. Assoc. 302, 627-637. doi: 10.1001/jama.2009.1144

Schölkopf, B., and Smola, A. (2002). Learning with Kernels: Support Vector Machines, Regularization, Optimization, and Beyond. Cambridge, MA: MIT Press.

Sluimer, J. D., Van Der Flier, W. M., Karas, G. B., Van Schijndel, R., Barnes, J., Boyes, R. G., et al. (2009). Accelerating regional atrophy rates in the progression from normal aging to Alzheimer's disease. Eur. Radiol. 19, 2826-2833. doi: 10.1007/s00330-009-1512-5

Snowdon, D. A. (2003). Healthy aging and dementia: findings from the Nun Study. Ann. Intern. Med. 139, 450-454. doi: 10.7326/0003-4819-139-5_Part_2200309021-00014

Solfrizzi, V., Capurso, C., D'introno, A., Colacicco, A. M., Santamato, A., Ranieri, M., et al. (2008). Lifestyle-related factors in predementia and dementia syndromes. Expert Rev. Neurother. 8, 133-158. doi: 10.1586/14737175. 8.1.133

Spulber, G., Niskanen, E., Macdonald, S., Smilovici, O., Chen, K., Reiman, E. M., et al. (2010). Whole brain atrophy rate predicts progression from MCI to Alzheimer's disease. Neurobiol. Aging 31, 1601-1605. doi: 10.1016/j.neurobiolaging.2008.08.018

Steele, M., Stuchbury, G., and Munch, G. (2007). The molecular basis of the prevention of Alzheimer's disease through healthy nutrition. Exp. Gerontol. 42, 28-36. doi: 10.1016/j.exger.2006.06.002

Taki, Y., Kinomura, S., Sato, K., Inoue, K., Goto, R., Okada, K., et al. (2008). Relationship between body mass index and gray matter volume in 1,428 healthy individuals. Obesity (Silver Spring) 16, 119-124. doi: 10.1038/oby. 2007.4

Terribilli, D., Schaufelberger, M. S., Duran, F. L., Zanetti, M. V., Curiati, P. K., Menezes, P. R., et al. (2011). Age-related gray matter volume changes in the brain during non-elderly adulthood. Neurobiol. Aging 32, 354-368. doi: 10.1016/j.neurobiolaging.2009.02.008

Tipping, M. E. (2001). Sparse bayesian learning and the relevance vector machine. J. Mach. Learn. Res. 1, 211-244. doi: 10.1162/15324430152748236

Tohka, J., Zijdenbos, A., and Evans, A. (2004). Fast and robust parameter estimation for statistical partial volume models in brain MRI. Neuroimage 23, 84-97. doi: 10.1016/j.neuroimage.2004.05.007

Wang, P. N., Liu, H. C., Lirng, J. F., Lin, K. N., and Wu, Z. A. (2009). Accelerated hippocampal atrophy rates in stable and progressive amnestic mild cognitive impairment. Psychiatry Res. 171, 221-231. doi: 10.1016/j.pscychresns.2008.05.002

Wilke, M., Schmithorst, V. J., and Holland, S. K. (2003). Normative pediatric brain data for spatial normalization and segmentation differs from standard adult data. Magn. Reson. Med. 50, 749-757. doi: 10.1002/mrm.10606

Xu, G., Liu, X., Yin, Q., Zhu, W., Zhang, R., and Fan, X. (2009). Alcohol consumption and transition of mild cognitive impairment to dementia. Psychiatry Clin. Neurosci. 63, 43-49. doi: 10.1111/j.1440-1819.2008.01904.x

Zylberstein, D. E., Lissner, L., Bjorkelund, C., Mehlig, K., Thelle, D. S., Gustafson, D., et al. (2011). Midlife homocysteine and late-life dementia in women. A prospective population study. Neurobiol. Aging 32, 380-386. doi: 10.1016/j.neurobiolaging.2009.02.024

Conflict of Interest Statement: The authors declare that the research was conducted in the absence of any commercial or financial relationships that could be construed as a potential conflict of interest.

Received: 31 October 2013; accepted: 28 April 2014; published online: 23 May 2014. Citation: Franke K, Ristow M and Gaser C (2014) Gender-specific impact of personal health parameters on individual brain aging in cognitively unimpaired elderly subjects. Front. Aging Neurosci. 6:94. doi: 10.3389/fnagi.2014.00094

This article was submitted to the journal Frontiers in Aging Neuroscience.

Copyright () 2014 Franke, Ristow and Gaser. This is an open-access article distributed under the terms of the Creative Commons Attribution License (CC BY). The use, distribution or reproduction in other forums is permitted, provided the original author(s) or licensor are credited and that the original publication in this journal is cited, in accordance with accepted academic practice. No use, distribution or reproduction is permitted which does not comply with these terms. 